\title{
Linearized dispersion relations in viscous relativistic hydrodynamics
}

\author{
Guillermo Perna $\odot^{*}$ and Esteban Calzetta $\odot^{\dagger}$ \\ Departamento de Física, Facultad de Ciencias Exactas y Naturales, \\ Universidad de Buenos Aires and IFIBA, CONICET, Ciudad Universitaria, Buenos Aires 1428, Argentina
}

(Received 7 August 2021; accepted 5 October 2021; published 8 November 2021)

\begin{abstract}
We compute the dispersion relations for scalar, vector and tensor modes of a viscous relativistic fluid, linearized around an equilibrium solution, for a divergence type theory (which, in the linearized theory, includes Israel-Stewart theory and anisotropic hydrodynamics as particular cases) and contrast them to the corresponding results derived from kinetic theory under the relaxation time approximation, and from causal first order theories. We conclude that all approaches give similar dynamics for the scalar and vector modes, while the particular divergence type theory presented here also contains propagating damped tensor waves, in agreement with kinetic theory. Nonhydrodynamic tensor modes are also a feature of holographic fluids. These results support the application of hydrodynamics in problems involving the interaction between fluids and gravitational waves.
\end{abstract}

DOI: 10.1103/PhysRevD.104.096005

\section{INTRODUCTION}

Recent developments in relativistic heavy ion collisions [1] and cosmology [2] have brought attention to the physics of relativistic viscous fluids [3,4], particularly since the realization that hydrodynamical models act as an attractor to more complex physics even on short times scales [5-11]. However, progress has been impaired by the fact that, unless the situation for nonrelativistic fluids described by the Navier-Stokes equations, no single approach to relativistic viscous fluids has achieved consensus status in the community. This is not a matter of "right" vs "wrong" but rather that different approaches best capture some aspects of the complex physics of relativistic fluids.

Given this situation, it is important to develop tests where the predictions of different approaches may be contrasted, thereby helping to select the most adequate choice for a given physical problem. One strategy that has been extensively used in the literature is to apply different approaches to a problem which could also be solved using a more fundamental theory. In this sense, the Bjorken and Gubser models of the expanding fireball in a relativistic heavy ion collision have been a preferred choice [12-15]. In this case the more fundamental theory is kinetic theory

guillermoezequielperna@gmail.com

calzetta@df.uba.ar

Published by the American Physical Society under the terms of the Creative Commons Attribution 4.0 International license. Further distribution of this work must maintain attribution to the author(s) and the published article's title, journal citation, and DOI. Funded by SCOAP ${ }^{3}$. under the relaxation time approximation [16-19], or else holographic fluids in an asymptotic region [20].

Another approach is to consider linearized perturbations of an equilibrium state, and to identify the propagating modes and their dispersion relations. Dispersion relations are known from kinetic theory under the relaxation time approximation [1,21-24]. They may be found also from quantum field theories, in the weakly coupled limit trough a perturbative expansion in the coupling constant [25], or else in the infinite coupling limit for holographic fluids [1,20,26-35]. All these approaches give similar though not identical results. Our goal is to contrast these "first principles" dispersion relations to the ones obtained from hydrodynamics.

We shall consider only conformal theories with no conserved charges. Then the focus of interest of a hydrodynamic model is the energy-momentum tensor (EMT) $T^{\mu \nu}$, which satisfies the conservation law

$$
T_{; \nu}^{\mu \nu}=0 .
$$

There is also an entropy flux $S^{\mu}$ that satisfies the "second law"

$$
S_{; \mu}^{\mu}=\Sigma
$$

where $\Sigma \geq 0$ is the entropy production.

For an ideal fluid

$$
T_{\text {ideal }}^{\mu \nu}=\rho u^{\mu} u^{\nu}+p \Delta^{\mu \nu},
$$

where $\rho$ is the energy density, $u^{\mu}$ is the velocity, restricted to the shell $u^{2}=-1$ (we work with signature $(-,+,+,+$ ) 
and natural units $\left.\hbar=k_{B}=c=1\right), \Delta^{\mu \nu}=g^{\mu \nu}+u^{\mu} u^{\nu}$ and $p$ is the pressure. For a conformal fluid $T^{\mu \nu}$ must be traceless, $T_{\mu}^{\mu}=0$, and so $p=\rho / 3$. Since there are no conserved charges and therefore no chemical potentials we may define the entropy density $s$ and the temperature $T$ through

$$
\frac{\partial p}{\partial T}=s=\frac{p+\rho}{T} .
$$

Then $\rho=\sigma T^{4}$, where $\sigma$ is a constant, $s=4 \sigma T^{3} / 3$, $S^{\mu}=s u^{\mu}$ and $\Sigma=0$.

In equilibrium the EMT of any fluid takes the ideal form (3) [36]. Thereby in a weakly nonequilibrium state it is natural to write

$$
T^{\mu \nu}=T_{0}^{\mu \nu}+\Pi^{\mu \nu},
$$

where $T_{0}^{\mu \nu}$ has the ideal form (3) and $\Pi^{\mu \nu}$ describes the viscous effects. For a conformal fluid $\Pi^{\mu \nu}$ must be traceless.

The problem is that now the four equations (1) are not enough to describe the evolution of the ten components of the symmetric tensor $T^{\mu \nu}$. This leads to two large families of theories. In the so-called first order theories (FOTs), constitutive relations are provided which restrict $\Pi^{\mu \nu}$ to be a given function of $T, u^{\mu}$ and their derivatives, thus keeping the number of degrees of freedom down to four. The socalled second order theories (SOTs), on the other hand, regard $\Pi^{\mu \nu}$, or a set of "nonequilibrium tensors" from which $\Pi^{\mu \nu}$ may be computed, as dynamical variables in their own right, and provide supplementary equations of motion.

Historically the first approaches to relativistic viscous fluids have been the Eckart and Landau-Lifshitz ones, which belong to the FOT class [37,38]. They provide covariant generalizations of the Navier-Stokes equations. They differ from each other in the definition of the fluid velocity $u^{\mu}$, which in the Eckart approach is defined from the flux of a conserved charge, while in the Landau-Lifshitz approach is defined form the energy flux, namely

$$
T^{\mu \nu} u_{\nu}=-\rho u^{\mu},
$$

which also defines $\rho$. Since the velocity appears in the constitutive relations for the viscous tensor $\Pi^{\mu \nu}$, the difference between the Eckart and Landau-Lifshitz theories is not just a matter of a choice of "frame," they actually are different theories [39]. Since in this paper we shall only consider conformally invariant theories with no conserved charges, it is natural to restrict ourselves to the LandauLifshitz approach, to be discussed in more detail below.

These first generation FOTs were proven to violate causality and to have no stable solutions [40-48]. However, it has been claimed that first order theories may be causal and stable if more general constitutive relations are considered [14,49-57]. This claim has gained considerable attention, since first order theories are generally simpler that second order ones, and in particular easier to implement numerically [58].

Concerning more general approaches such as BaierRomatschke-Son-Starinets-Stephanov [1,20,59] and third order hydrodynamics $[60,61]$, which consists of writing the most general form for the energy-momentum tensor containing terms with up to a certain number of derivatives restricted by conformal invariance, the issue is whether the viscous energy momentum tensor is restricted to be proportional to the shear tensor, or else regarded as a hydrodynamic variable on its own. In the first case we obtain a theory within the FOT class, while in the second it becomes a SOT.

The problem with the Eckart and Landau-Lifshitz approaches may be tracked down to the fact that, when defining the entropy production, some second order terms were retained while others were arbitrarily rejected [36]. This problem may be solved (or at least alleviated) by enlarging the set of degrees of freedom of the theory, and likewise introducing new terms in the entropy production. This leads to SOT approaches, such as Israel-Stewart [62-67], extended thermodynamics [68-70], Denicol-Niemi-Molnár-Rischke [71-79], anisotropic hydrodynamics [80-87], and divergence type theories (DTTs) [88-98]. We shall focus on a particular implementation of the DTT paradigm, which, at the linearized level, contains the others as particular cases.

To the best of our knowledge, both FOTs and SOTs have been tested in Bjorken and Gubser backgrounds, where they successfully reproduce the results from kinetic theory under the relaxation time approximation $[14,15,99]$.

In this paper we will consider a conformal fluid in an equilibrium state in Minkowski space-time, and compute the response of the EMT to a perturbation in the metric, assuming the dynamics is described by a DTT to be presented below. We shall compare the result with the same quantity as derived from a FOT, and from kinetic theory under the relaxation time approximation. We shall comment briefly on the corresponding result for quantum fields $[1,20,25-35]$. In other words, we shall compute the propagator

$$
G^{\mu \nu \rho \sigma}\left[x, x^{\prime}\right]=\left.\frac{\delta T^{\mu \nu}[x]}{\delta g_{\rho \sigma}\left[x^{\prime}\right]}\right|_{g_{\mu \nu}=\eta_{\mu \nu}}
$$

The poles of the propagator as we approach the infrared limit indicate the propagating modes in the hydrodynamic limit and their dispersion relations.

The rest of the paper is organized as follows. In the next section we summarize the well-known dispersion relations from kinetic theory [21-24] (Sec. II). Then in the following section we compute the EMT response in a divergence type theory (Sec. III). We present some brief conclusions in the final section. 
For completeness we also present the relevant dispersion relations for ideal and Landau-Lifshitz fluids and causal FOTs [14] in Appendix A.

\section{DISPERSION RELATIONS FROM KINETIC THEORY}

In this section we shall derive the dispersion relations from kinetic theory. We observe that the kinetic theory EMT propagators are computed in closed form in Refs. [21-24]. They display a complex analytic structure dominated by branch cuts. However, in the asymptotic regime where hydrodynamics is expected to hold, this analytic structure may be mimicked by a suitable distribution of poles. Our interest lies in finding these equivalent poles in the $k^{2} \rightarrow 0$ limit, both the hydrodynamic ones and the longest living nonhydrodynamic modes.
We consider an equilibrium state in Minkowski spacetime, whereby the metric $g_{\mu \nu}=\eta_{\mu \nu}=\operatorname{diag}(-1,1,1,1)$, the velocity $U^{\mu}$ and the temperature $T_{0}$ are constant. The EMT $T_{0}^{\mu \nu}$ takes the ideal form Eq. (3). Without a loss of generality we may assume $U^{\mu}=(1,0,0,0)$. We consider a fluctuation in the metric $\eta_{\mu \nu} \rightarrow g_{\mu \nu}=\eta_{\mu \nu}+h_{\mu \nu}$, and the corresponding change in the EMT $T^{\mu \nu}=T_{0}^{\mu \nu}+\delta T^{\mu \nu}$. Linearizing with respect to $h_{\mu \nu}$ we may read the propagator Eq. (7) from the relationship

$$
\delta T^{\mu \nu}[x]=\int d^{4} x^{\prime} G^{\mu \nu \rho \sigma}\left[x, x^{\prime}\right] h_{\rho \sigma}\left[x^{\prime}\right] .
$$

Four of these relationships are trivial, since they correspond to coordinate changes. If $x^{\mu} \rightarrow x^{\prime \mu}=x^{\mu}+\xi^{\mu}$, then $h_{\mu \nu}=-\xi_{\mu, \nu}-\xi_{\nu, \mu}$, and $\delta T^{\mu \nu}=\xi_{, \rho}^{\mu} T_{0}^{\rho \nu}+\xi_{, \rho}^{\nu} T_{0}^{\rho \mu}$. So, using $\Delta_{0}^{\mu \nu}=\eta^{\mu \nu}+U^{\mu} U^{\nu}$ we get

$$
\rho_{0}\left[\dot{\xi}^{\mu} U^{\nu}+\dot{\xi}^{\nu} U^{\mu}+\frac{1}{3}\left(\Delta_{0}^{\mu \rho} \xi_{, \rho}^{\nu}+\Delta_{0}^{\nu \rho} \xi_{, \rho}^{\mu}\right)\right]=-2 \int d^{4} x^{\prime} G_{, \sigma}^{\mu \nu \rho \sigma}\left[x, x^{\prime}\right] \xi_{\rho}\left[x^{\prime}\right]
$$

with $\dot{\xi}^{\mu}=U^{\nu} \xi_{; \nu}^{\mu}$, so

$$
G_{, \sigma}^{\mu \nu \rho \sigma}\left[x, x^{\prime}\right]=\frac{1}{2} \rho_{0}\left[\eta^{\mu \rho}\left(U^{\nu} U^{\lambda}+\frac{1}{3} \Delta_{0}^{\nu \lambda}\right)+\eta^{\nu \rho}\left(U^{\mu} U^{\lambda}+\frac{1}{3} \Delta_{0}^{\mu \lambda}\right)\right] \partial_{\lambda} \delta\left(x-x^{\prime}\right) .
$$

Since the background is homogeneous, we expect the propagators to be translation invariant. Then we may Fourier transform

$$
G^{\mu \nu \rho \sigma}=\int \frac{d \omega d^{3} k}{(2 \pi)^{4}} e^{i\left[\mathbf{k} \cdot\left(\mathbf{x}-\mathbf{x}^{\prime}\right)-\omega\left(t-t^{\prime}\right)\right]} G^{\mu \nu, \rho \sigma}[\mathbf{k}, \omega],
$$

whereby

$$
i \omega G^{\mu \nu \rho 0}[\mathbf{k}, \omega]=i k_{j} G^{\mu \nu \rho j}[\mathbf{k}, \omega]-\frac{i}{2} \rho_{0}\left[\eta^{\mu \rho}\left(U^{\nu} \omega-\frac{1}{3} \Delta_{0}^{\nu j} k_{j}\right)+\eta^{\nu \rho}\left(U^{\mu} \omega-\frac{1}{3} \Delta_{0}^{\mu j} k_{j}\right)\right] .
$$

This means that $G^{\mu \nu \rho 0}$ is trivially obtained from $G^{\mu \nu, j k}$, and so there is no loss of generality in computing the propagators under the gauge condition $h_{\mu 0}=0$.

On general grounds we expect the propagators will obey the reciprocity condition $[100,101]$

$$
G^{\mu \nu \rho \sigma}\left[x, x^{\prime}\right]=G^{\rho \sigma \mu \nu}\left[x^{\prime}, x\right],
$$

whereby we also do not need to compute explicitly the propagators of the form $G^{0 \nu \rho \sigma}$. So the only nontrivial problem is to compute the variation of $T^{i j}$ upon a metric fluctuation $h_{i j}$. However, to complete this task we need some information on the fundamental degrees of freedom of the theory and their dynamics.

In kinetic theory $[3,15,102]$, the fundamental description is provided by the distribution function $f\left(x^{\mu}, p_{\mu}\right)$, where $p^{\mu}$, for a conformal theory, is restricted to the future light cone $p^{2}=0, p^{0} \geq 0 . f$ obeys the Boltzmann equation

$$
p^{\mu} f_{; \mu}=I_{\mathrm{col}}[f] \text {, }
$$

where the phase space covariant derivative is

$$
f_{; \mu}=\frac{\partial f}{\partial x^{\mu}}+\Gamma_{\mu \rho}^{\nu} p_{\nu} \frac{\partial f}{\partial p_{\rho}} .
$$

Covariant derivatives are taken with the first order connection

$$
\Gamma_{\nu \lambda}^{\mu}=\frac{1}{2} \eta^{\mu \rho}\left[h_{\nu \rho, \lambda}+h_{\lambda \rho, \nu}-h_{\nu \lambda, \rho}\right]
$$

Observe that $\Gamma_{\nu \lambda}^{\nu}=h_{i, \lambda}^{i} / 2$ as expected, and $\Gamma_{\nu \lambda}^{\mu} U^{\nu} U^{\lambda}=0$. 
For simplicity, we shall only consider the MaxwellJüttner case where the equilibrium distribution has the form

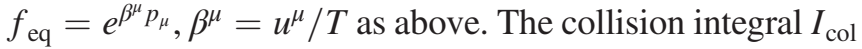
vanishes in equilibrium. It also has to satisfy the constraint

$$
\int D p p^{\mu} I_{\mathrm{col}}[f]=0
$$

with the phase space covariant measure

$$
D p=\frac{4 \pi d^{4} p_{\mu}}{(2 \pi)^{4} \sqrt{-g}} \delta\left(-p^{2}\right) \theta\left(p^{0}\right),
$$

which enforces conservation Eq. (1) for the EMT

$$
T^{\mu \nu}[f]=\int D p p^{\mu} p^{\nu} f
$$

and the $H$ theorem

$$
H=\int D p \ln [f] I_{\mathrm{col}}[f] \leq 0
$$

for any solution of the Boltzmann equation (14). Validity of the $H$ theorem (20) enforces the second law (2) with the entropy flux

$$
S^{\mu}[f]=\int D p p^{\mu} f[1-\ln f]
$$

and entropy production $\Sigma=-H$.

To a given $f$ we may associate an EMT [Eq. (19)] and thereby a velocity $u^{\mu}$ and an energy density $\rho \equiv \sigma T^{4}$ through the Landau-Lifshitz prescription (6). We then adopt the relaxation time approximation [16-19]

$$
I_{\mathrm{col}}=\frac{1}{\tau} u^{\mu} p_{\mu}\left[f-f_{\mathrm{eq}}\right]
$$

where $f_{\text {eq }}$ is the Maxwell-Jüttner distribution with the same $T$ and $u^{\mu}$ as $f$. The constant $\tau$ is the so-called relaxation time.

In equilibrium $T=T_{0}, u^{\mu}=U^{\mu}=(1,0,0,0), g^{\mu \nu}=\eta^{\mu \nu}$ and $f=f_{0}=f_{\text {eq }, 0}=e^{-p^{0} / T_{0}}$. We consider a metric fluctuation $g_{\mu \nu}=\eta_{\mu \nu}+h_{\mu \nu}$ with $h_{0 \nu}=0$. Subsequently we have $T=T_{0}(1+\vartheta)$ and $u^{\mu}=U^{\mu}+v^{\mu}$ with $v^{0}=0$. We parametrize

$$
f=f_{0}\left[1+\vartheta \frac{p^{0}}{T_{0}}+v^{k} \frac{p_{k}}{T_{0}}+\varphi\right]
$$

where $\vartheta$ and $v^{\mu}$ are the perturbations to the Landau-Lifshitz temperature and velocity, namely $\int \frac{d^{3} p}{(2 \pi)^{3} p}\left(U_{\nu}+v_{\nu}\right) p^{\nu} p^{\mu} f=-\sigma T_{0}^{4}\left[(1+4 \vartheta) U^{\mu}+v^{\mu}\right]$,

which is equivalent to

$$
\int \frac{d^{3} p}{(2 \pi)^{3}} p^{\mu} \varphi f_{0}=0
$$

Then also

$$
f_{\text {eq }}=f_{0}\left[1+\vartheta \frac{p^{0}}{T_{0}}+v^{k} \frac{p_{k}}{T_{0}}\right] .
$$

To perform the scalar-vector-tensor decomposition we write

$$
v_{i}=i k_{i} v_{S}+v_{V i},
$$

with $k_{j} v_{V j}=0$, and

$$
\begin{aligned}
h_{i j}= & {\left[k_{i} k_{j}-k^{2} \delta_{i j}\right] h_{S}+\left[k_{i} k_{j}-\frac{1}{3} \delta_{i j} k^{2}\right] h_{S}^{\prime} } \\
& +i k_{i} h_{V j}+i k_{j} h_{V i}+h_{T i j},
\end{aligned}
$$

with $k_{j} h_{V j}=k_{j} h_{T i j}=h_{T j j}=0 . \vartheta, v_{S}, h_{S}$ and $h_{S}^{\prime}$ correspond to scalar degrees of freedom, $v_{V}$ and $h_{V}$ are vector degrees of freedom, and $h_{T}^{j k}$ are the tensor degrees of freedom. The Boltzmann equation (14) becomes

$$
\begin{aligned}
& \frac{p}{T_{0}}\left(p \vartheta_{, t}+p^{k} \vartheta_{, k}\right)+\frac{p_{j}}{T_{0}}\left(p v_{, t}^{j}+p^{k} v_{, k}^{j}\right)+p \varphi_{, t}+p^{k} \varphi_{, k} \\
& \quad+\frac{1}{2 T_{0}} \dot{h}_{j k} p^{j} p^{k}=\frac{-p}{\tau} \varphi .
\end{aligned}
$$

Fourier transforming the space-time dependence, we get

$$
\varphi=\frac{p}{T_{0}} \frac{\left[-\frac{\omega}{k}+\hat{k}_{l} \hat{p}^{l}\right]\left(\vartheta+\hat{p}_{j} v_{j}\right)-\frac{\omega}{2 k} h_{j k} \hat{p}^{j} \hat{p}^{k}}{z-\hat{k}_{l} \hat{p}^{l}},
$$

where

$$
z=\frac{1}{k}\left[\omega+i \tau^{-1}\right] .
$$

On the other hand, if we multiply Eq. (58) by $f_{0}$ and integrate over all momenta, the terms containing $\varphi$ cancel because of Eq. (25), and we get the continuity equation

$$
4 \sigma T_{0}^{4}\left\{-i \omega \vartheta-\frac{1}{3} k^{2} v_{S}+\frac{i}{3} \omega k^{2} h_{S}\right\}=0 .
$$

We still must enforce the constraints in Eq. (25), which become 


$$
\begin{gathered}
{\left[-\frac{\omega}{k} J+\hat{k}_{l} J^{l}\right] \vartheta+\left[-\frac{\omega}{k} J^{k}+\hat{k}_{l} J^{l k}\right] v_{k}=\frac{\omega}{2 k} h_{j k} J^{j k},} \\
{\left[-\frac{\omega}{k} J^{j}+\hat{k}_{l} J^{j l}\right] \vartheta+\left[-\frac{\omega}{k} J^{j k}+\hat{k}_{l} J^{j l k}\right] v_{k}=\frac{\omega}{2 k} h_{l k} J^{j k l},}
\end{gathered}
$$

where

$$
J^{k_{1} \ldots k_{n}}=\int \frac{d^{3} p}{(2 \pi)^{3}} \frac{p^{2} \hat{p}^{k_{1}} \ldots \hat{p}^{k_{n}}}{z-\hat{k}_{l} \hat{p}^{l}} e^{-p / T_{0}}
$$

They are evaluated in Appendix B. After finding $\vartheta, v^{k}$ and $\varphi$, we may proceed to compute the EMT

$$
\begin{aligned}
T^{\mu \nu}= & \rho T_{0}^{4}(1+4 \vartheta)\left[U^{\mu} U^{\nu}+\frac{1}{3} \Delta^{\mu \nu}+U^{\mu} v^{\nu}+U^{\nu} v^{\mu}\right] \\
& +\Pi^{\mu \nu}
\end{aligned}
$$

where

$$
\Pi^{\mu \nu}=\int \frac{d^{3} p}{(2 \pi)^{3} p} p^{\nu} p^{\mu} \varphi f
$$

or else

$$
\begin{aligned}
\Pi^{j k}= & \frac{1}{T_{0}}\left\{\left[-\frac{\omega}{k} J^{j k}+\hat{k}_{l} J^{j k l}\right] \vartheta+\left[-\frac{\omega}{k} J^{j k m}+\hat{k}_{l} J^{j k l m}\right] v_{m}\right. \\
& \left.-\frac{\omega}{2 k} h_{l m} J^{j k l m}\right\} .
\end{aligned}
$$

Observe that the trace of $\Pi^{j k}$ vanishes because the $J^{k_{1} \ldots k_{n}}$ tensors obey $J_{j}^{j k_{1} \ldots k_{n}}=J^{k_{1} \ldots k_{n}}$.

It is clear that the scalar, vector and tensor sectors decouple, and it is best to consider each one in turn. We expect to recover the Landau-Lifshitz hydrodynamic modes from divergences in $\vartheta$ and $v^{j}$, while there will be poles in $\varphi$ associated to nonhydrodynamic modes absent from Landau-Lifshitz theory.

\section{A. Hydrodynamic poles}

\section{The scalar sector}

In the scalar sector we have

$$
h_{i j}=\left[k_{i} k_{j}-k^{2} \delta_{i j}\right] h_{S}+\left[k_{i} k_{j}-\frac{1}{3} \delta_{i j} k^{2}\right] h_{S}^{\prime}
$$

and $v^{k}=i v_{S} k^{k}$. From the results of Appendix B we get

$$
\begin{aligned}
& {\left[-\frac{\omega}{k} J+\chi_{1}\right] \vartheta+\left[-\frac{\omega}{k} \chi_{1}+\frac{2}{3} \chi_{3}\right] i k v_{S}} \\
& =\frac{\omega k}{3}\left[\left(-\frac{3}{2} J+\chi_{3}\right) h_{S}+\left(-\frac{1}{2} J+\chi_{3}\right) h_{S}^{\prime}\right], \\
& \left.=\frac{\omega k}{k} \chi_{1}+\frac{2}{3} \chi_{3}\right] \vartheta+\left[-\frac{2}{3} \frac{\omega}{k} \chi_{3}+\frac{2}{5} \chi_{5}\right] i k v_{S} \\
& \left.=\left(2 \chi_{5}-5 \chi_{1}\right) h_{S}+\left(2 \chi_{5}-\frac{5}{3} \chi_{1}\right) h_{S}^{\prime}\right] .
\end{aligned}
$$

We expect to find the hydrodynamic poles when the determinant of this system vanishes. We consider the $\tau \rightarrow 0$ limit, where $|z| \gg 1$. The condition for a vanishing determinant, to next to lowest order in $z^{-1}$, is

$$
\omega \approx \pm \frac{k}{\sqrt{3}}-\frac{2}{15} i \tau k^{2}
$$

which reproduces the result for Landau-Lifshitz fluids [Eq. (A11)], identifying $\nu=\tau / 5$.

\section{The vector sector}

In the vector sector

$$
h_{i j}=i k_{i} h_{V j}+i k_{j} h_{V i}
$$

with $h_{V i} k^{i}=0$. Then $\vartheta=0$ and $v^{i}=v_{V}^{i}$ is also transverse. We find

$\left[-\frac{\omega}{k}\left(\frac{1}{2} J-\frac{1}{3} \chi_{3}\right)+\frac{1}{2} \chi_{1}-\frac{1}{5} \chi_{5}\right] v_{V}^{k}=\frac{i \omega}{2}\left(\chi_{1}-\frac{2}{5} \chi_{5}\right) h_{V}^{k}$.

There is a pole when the term in brackets in the left-hand side vanishes. For large $z$ [cf. Eq. (31)] we find the pole at

$$
\omega \approx \frac{1}{5} \frac{-i k^{2} \tau}{(1-\omega \tau)}
$$

which corresponds to the hydrodynamic pole we find in Landau-Lifshitz fluids, again identifying $\nu=\tau / 5$ [cf. Eq. (A10)].

In the tensor sector $h_{i j}=h_{T i j}$ is both traceless and divergenceless, $\vartheta=v^{i}=0$, and there are no hydrodynamic poles.

\section{B. Nonhydrodynamic poles}

To study the nonhydrodynamic modes, we shall consider $\Pi^{j k}$ in the limit where $k \rightarrow 0$, while $\omega$ goes to a finite value and $|z| \gg 1$. Once again, it is best to consider scalar, vector and tensor modes separately. 


\section{Scalar modes}

In the limit we are considering, the scalar viscous EMT reads

$$
\Pi_{S}^{j k}=\frac{-\omega}{T_{0} k}\left\{J^{j k} \vartheta+J^{j k m} \hat{k}_{m}\left(i k v_{S}\right)+\frac{1}{2} k^{2}\left[\left(h_{S}+h_{S}^{\prime}\right) J^{j k l m} \hat{k}_{l} \hat{k}_{m}-\left(h_{S}+\frac{1}{3} h_{S}^{\prime}\right) J^{j k l m} \delta_{l m}\right]\right\} .
$$

Since $\Pi_{S j}^{j}=0$ we may write

$$
\begin{gathered}
\Pi_{S}^{j k}=\Pi_{S}\left[\hat{k}^{j} \hat{k}^{k}-\frac{1}{3} \delta^{j k}\right], \\
\Pi_{S}=\frac{3}{2} \Pi_{S}^{j k} \hat{k}_{j} \hat{k}_{k},=\frac{-\omega}{T_{0} k}\left\{\chi_{3} \vartheta+\frac{3}{5} \chi_{5}\left(i k v_{S}\right)+\frac{1}{2} k^{2}\left[\left(h_{S}+h_{S}^{\prime}\right)\left(-\frac{3}{2} J+2 \chi_{3}+12 \chi_{6}\right)-\left(h_{S}+\frac{1}{3} h_{S}^{\prime}\right) \chi_{3}\right]\right\} .
\end{gathered}
$$

It may be seen that to this order there are no new poles proportional to $h_{S}$, so we shall set $h_{S}=0$. Then we only need the leading order form of $i k v_{S}$ and $\vartheta$. In the scalar case Eq. (32) and the two equations (33) are not independent, we choose to work with the former and the first of the latter. Then

$$
i k v_{S}=-\frac{2 k^{2}}{15 z} h_{S}^{\prime}
$$

while $\vartheta$ is of higher order in $k$. From Eq. (46) we now find

$$
\Pi_{S}=-\frac{\sigma T_{0}^{4} \omega k}{z} \frac{4}{15}\left(1+\frac{9}{35} \frac{1}{z^{2}}\right) h_{S}^{\prime}
$$

We see that the leading order terms in the inverse propagator go like $1-\left(9 / 35 z^{2}\right)$. This behavior may be reproduced by a nonhydrodynamic pole at $z^{2}=9 / 35$, or else

$$
\omega=\frac{-i}{\tau} \pm \sqrt{\frac{9}{35}} k .
$$

\section{Vector modes}

As with the scalar modes, we begin with the leading order relation

$$
v_{V}^{k} \approx \frac{-i k}{5 z} h_{V}^{k}
$$

The vector viscous EMT reduces to

$$
\Pi_{V}^{j k}=\frac{-4 i \omega}{15 z T_{0}}\left\{1+\frac{8}{35} \frac{1}{z^{2}}\right\}\left(\hat{k}^{j} h_{V}^{k}+\hat{k}^{k} h_{V}^{j}\right) .
$$

Therefore the leading terms in the inverse propagator are $\propto 1-(8 / 35) z^{-2}$, "as if" there were a nonhydrodynamic pole at

$$
\omega=-\frac{i}{\tau} \pm \sqrt{\frac{8}{35}} k
$$

\section{Tensor modes}

The response of the EMT to a tensor metric fluctuation is

$$
\Pi_{T}^{j k}=\frac{-\omega}{T_{0} k} \chi_{6} h_{T}^{j k} .
$$

For large $z$ we get

$$
\Pi_{T}^{j k}=\frac{-4 i \omega \tau}{15(1-i \omega \tau)} \sigma T_{0}^{4} h_{T}^{j k}\left[1+\frac{1}{7} \frac{1}{z^{2}}+\ldots\right] .
$$

Therefore the inverse propagator will be proportional to $1-1 / 7 z^{2}$, which is the behavior caused by a pole at [21]

$$
\omega=-\frac{i}{\tau} \pm \frac{k}{\sqrt{7}} .
$$

We see from kinetic theory that a relativistic fluid may support damped tensor waves, which are totally absent in first order theories.

\section{Poles or cuts?}

The analytic structure of the kinetic theory propagators is determined by the presence of a cut in the complex frequency plane. This is due to the fact that the propagators depend on the function $L[z]$ defined in Eq. (B2), which has a cut from $z=-1$ to $z=1$ or else, with $z$ as in Eq. (31), from $\omega=-i / \tau-k$ to $-i / \tau+k$ [22]. However, the analytic structure of the propagators in the $k \rightarrow 0$ limit may be reproduced by suitably locating poles in the complex $\omega$ plane. Though the poles themselves are not in the asymptotic region, they give the right analytic structure there.

Coincidentally, the analytic structure of EMT correlators in $\lambda \phi^{4}$ theory is determined by cuts, rather than poles [25].

However, in the strong coupling limit things seem to be different. We can verify this explicitly in theories with a holographic dual [1,20,26-35]. Then the thermal EMT correlators may be found by solving the classical Einstein equations in a dual, five dimensional space-time. A thermal state corresponds to an anti-de Sitter space-time containing 
a black hole in its center. The physical fluid lives on the boundary of the anti-de Sitter space-time, and the Hawking temperature of the hole is the temperature of the fluid. Perturbations of the fluid correspond to perturbations of the black hole metric, with no naked singularities and incoming boundary conditions at the horizon.

For each $k$, the frequencies of free oscillations of the fluid correspond to the horizon fluctuations, and they come in a discrete, infinite series, depending on the t'Hooft coupling $g^{2} N_{c}$, where $g$ and $N_{c}$ are the coupling and number of colors of the underlying gauge theory [103]. These free frequencies appear as poles in the EMT propagators. For the scalar ("sound") and vector ("shear") sectors, there is sequence of hydrodynamic poles, namely $\omega \rightarrow 0$ when $k \rightarrow 0$ and also nonhydrodynamic poles. In the infinite coupling limit, the hydrodynamic poles are well reproduced by a FOT with $\nu=4 \pi / T_{0}$. Corrections to this value at finite coupling are discussed in [104]. In the tensor (in this context frequently called "scalar") sector, there are no hydrodynamic poles, but again an infinite, discrete sequence of nonhydrodynamic ones.

The presence of infinitely many modes cannot be reproduced by hydrodynamics, either first or second order. However, the imaginary part of the poles increases rather sharply along the sequence, and so only a few long lived modes are actually relevant to describe the approach to equilibrium. These are the modes that either causal FOTs or second order theories aim to reproduce.

\section{Beyond the relaxation time approximation}

While the relaxation time approximation we have used so far is frequently a preferred choice in view of its simplicity, it is also known [105] that it departs in significant ways from more realistic kinetic equations such as Boltzmann's and Landau's. It is therefore relevant to ask how far results derived under this approximation may be generalized to more complete setups, if at all.

To this end we adopt the viewpoint presented in [106]. We parametrize

$$
f=f_{0}(1+\phi)
$$

and

$$
I_{\text {col }}[f]=-\left(p^{0}\right) f_{0} \mathcal{F}[\phi] .
$$

The Boltzmann equation (14) becomes

$$
\phi_{, t}+\hat{p}^{k} \phi_{, k}+\frac{1}{2 T_{0} p^{0}} \dot{h}_{j k} p^{j} p^{k}=-\mathcal{F}[\phi] .
$$

We assume the collision term is ultralocal in position space. Then it commutes with Fourier transformation, and we get

$$
\left[\omega-k_{k} \hat{p}^{k}\right] \phi+\frac{\omega}{2 T_{0} p^{0}} h_{j k} p^{j} p^{k}=-i \mathcal{F}[\phi] .
$$

It is convenient to introduce an inner product in the space of functions of momentum

$$
\langle\psi \mid \phi\rangle=\int D_{\beta} p \psi^{*} \phi,
$$

where

$$
D_{\beta} p=D p p^{0} f_{0}
$$

We assume the linearized operator $\mathcal{F}$ is symmetric under this inner product. Momentum conservation takes the form

$$
\left\langle p^{\mu}|\mathcal{F}| \phi\right\rangle=0 .
$$

Since $|\phi\rangle$ can be any vector, it must be

$$
\mathcal{F}\left|p^{\mu}\right\rangle=0
$$

We assume these are the only null eigenvectors of the collision operator, and that there is a set of non-null eigenvectors

$$
\mathcal{F}\left|\phi_{\lambda}\right\rangle=\nu_{\lambda}\left|\phi_{\lambda}\right\rangle
$$

where $\nu_{\lambda}$ is real and positive, and $\left\langle\phi_{\sigma} \mid \phi_{\lambda}\right\rangle=\delta_{\sigma \lambda}$. We then have

$$
\begin{aligned}
|\phi\rangle & =\sum_{\mu=0}^{3} \alpha_{\mu}\left|p^{\mu}\right\rangle\left\langle p^{\mu} \mid \phi\right\rangle+\sum_{\lambda}\left|\phi_{\lambda}\right\rangle\left\langle\phi_{\lambda} \mid \phi\right\rangle, \\
\mathcal{F}|\phi\rangle & =\sum_{\lambda} \nu_{\lambda}\left|\phi_{\lambda}\right\rangle\left\langle\phi_{\lambda} \mid \phi\right\rangle,
\end{aligned}
$$

where (no sum over $\mu$ )

$$
\alpha_{\mu}=\left\langle p^{\mu} \mid p^{\mu}\right\rangle^{-1}
$$

Contracting Eq. (59) with the $\left|p^{\mu}\right\rangle$ or the $\left|\phi_{\lambda}\right\rangle$ we get two sets of equations. On one hand

$$
\sum_{\nu=0}^{3}\left[\omega \delta^{\mu \nu}-k_{k} \alpha_{\nu}\left\langle p^{\mu}\left|\hat{p}^{k}\right| p^{\nu}\right\rangle\right]\left\langle p^{\nu} \mid \phi\right\rangle-\sum_{\sigma} k_{k}\left\langle p^{\mu}\left|\hat{p}^{k}\right| \phi_{\sigma}\right\rangle\left\langle\phi_{\sigma} \mid \phi\right\rangle=-\frac{\omega}{2 T_{0}} h_{j k}\left\langle p^{\mu} \mid \frac{p^{j} p^{k}}{p^{0}}\right\rangle
$$


and on the other

$$
-\sum_{\nu=0}^{3} k_{k} \alpha_{\nu}\left\langle\phi_{\lambda}\left|\hat{p}^{k}\right| p^{\nu}\right\rangle\left\langle p^{\nu} \mid \phi\right\rangle+\sum_{\sigma}\left[\left(\omega+i \nu_{\sigma}\right) \delta_{\lambda \sigma}-k_{k}\left\langle\phi_{\lambda}\left|\hat{p}^{k}\right| \phi_{\sigma}\right\rangle\right]\left\langle\phi_{\sigma} \mid \phi\right\rangle=-\frac{\omega}{2 T_{0}} h_{j k}\left\langle\phi_{\lambda} \mid \frac{p^{j} p^{k}}{p^{0}}\right\rangle,
$$

where

$$
\begin{aligned}
\alpha_{0} & =\frac{1}{3} \alpha_{i}=2 \pi^{2}\left[T_{0}^{5} \Gamma[5]\right]^{-1}, \\
\left\langle p^{0}\left|\hat{p}^{k}\right| p^{0}\right\rangle & =\left\langle p^{j}\left|\hat{p}^{k}\right| p^{l}\right\rangle=\left\langle p^{l} \mid \frac{p^{j} p^{k}}{p^{0}}\right\rangle=0, \\
\left\langle p^{j}\left|\hat{p}^{k}\right| p^{0}\right\rangle & =\left\langle p^{0}\left|\hat{p}^{k}\right| p^{j}\right\rangle=\left\langle p^{0} \mid \frac{p^{j} p^{k}}{p^{0}}\right\rangle=\frac{T_{0}^{5} \Gamma[5]}{6 \pi^{2}} \delta^{j k} .
\end{aligned}
$$

We then get

$$
\begin{aligned}
\left\langle p^{0} \mid \phi\right\rangle & =\frac{k_{k}}{\omega}\left\langle p^{k} \mid \phi\right\rangle+\frac{k^{2} h_{S}}{3 T_{0} \alpha_{0}}, \\
\left\langle p^{i} \mid \phi\right\rangle & =\frac{k^{i}}{3 \omega}\left\langle p^{0} \mid \phi\right\rangle+\sum_{\sigma} \frac{k_{k}}{\omega}\left\langle\frac{p^{i} p^{k}}{p^{0}} \mid \phi_{\sigma}\right\rangle\left\langle\phi_{\sigma} \mid \phi\right\rangle, \\
\left\langle\phi_{\lambda} \mid \phi\right\rangle & =-\frac{\omega h_{j k}}{2 T_{0}\left(\omega+i \nu_{\lambda}\right)}\left\langle\phi_{\lambda} \mid \frac{p^{j} p^{k}}{p^{0}}\right\rangle+\frac{k_{k}}{\left(\omega+i \nu_{\lambda}\right)}\left\{\sum_{l} 3 \alpha_{0}\left\langle\phi_{\lambda} \mid \frac{p^{k} p^{l}}{p^{0}}\right\rangle\left\langle p^{l} \mid \phi\right\rangle+\sum_{\sigma}\left\langle\phi_{\lambda}\left|\hat{p}^{k}\right| \phi_{\sigma}\right\rangle\left\langle\phi_{\sigma} \mid \phi\right\rangle\right\} .
\end{aligned}
$$

Since we are only interested in the hydrodynamic limit, we may solve these equations in powers of $k^{j}$. To zeroth order

$$
\begin{aligned}
\left\langle p^{0} \mid \phi\right\rangle^{(0)} & =k^{2} h_{S} \frac{T_{0}^{4} \Gamma[5]}{6 \pi^{2}}, \\
\left\langle p^{j} \mid \phi\right\rangle^{(0)} & =0, \\
\left\langle\phi_{\lambda} \mid \phi\right\rangle^{(0)} & =-\frac{\omega h_{j k}}{2 T_{0}\left[\omega+i \nu_{\lambda}\right]}\left\langle\phi_{\lambda} \mid \frac{p^{j} p^{k}}{p^{0}}\right\rangle,
\end{aligned}
$$

where $\left\langle p^{0} \mid \phi\right\rangle$ receives no first order corrections. Else

$$
\begin{aligned}
& \left\langle p^{j} \mid \phi\right\rangle^{(1)}=\frac{1}{\omega}\left\{k^{j} k^{2} h_{S} \frac{T_{0}^{4} \Gamma[5]}{18 \pi^{2}}-\sum_{\sigma} k_{k}\left\langle\frac{p^{j} p^{k}}{p^{0}} \mid \phi_{\sigma}\right\rangle \frac{\omega h_{l m}}{2 T_{0}\left[\omega+i \nu_{\sigma}\right]}\left\langle\phi_{\sigma} \mid \frac{p^{l} p^{m}}{p^{0}}\right\rangle\right\}, \\
& \left\langle\phi_{\lambda} \mid \phi\right\rangle^{(1)}=-\frac{k_{k}}{\left(\omega+i \nu_{\lambda}\right)} \sum_{\sigma}\left\langle\phi_{\lambda}\left|\hat{p}^{k}\right| \phi_{\sigma}\right\rangle \frac{\omega h_{l m}}{2 T_{0}\left[\omega+i \nu_{\sigma}\right]}\left\langle\phi_{\sigma} \mid \frac{p^{l} p^{m}}{p^{0}}\right\rangle .
\end{aligned}
$$

To second order we get

$$
\begin{aligned}
\left\langle p^{0} \mid \phi\right\rangle^{(2)} & =\frac{k_{k}}{\omega}\left\langle p^{k} \mid \phi\right\rangle^{(1)}, \\
\left\langle p^{j} \mid \phi\right\rangle^{(2)} & =\frac{k_{k}}{\omega} \sum_{\sigma}\left\langle\frac{p^{j} p^{k}}{p^{0}} \mid \phi_{\sigma}\right\rangle\left\langle\phi_{\sigma} \mid \phi\right\rangle^{(1)}, \\
\left\langle\phi_{\lambda} \mid \phi\right\rangle^{(2)} & =\frac{k_{k}}{\left(\omega+i \nu_{\lambda}\right)}\left\{\sum_{l} \alpha_{l}\left\langle\phi_{\lambda} \mid \frac{p^{k} p^{l}}{p^{0}}\right\rangle\left\langle p^{l} \mid \phi\right\rangle^{(1)}+\sum_{\sigma}\left\langle\phi_{\lambda}\left|\hat{p}^{k}\right| \phi_{\sigma}\right\rangle\left\langle\phi_{\sigma} \mid \phi\right\rangle^{(1)}\right\} .
\end{aligned}
$$


Finally, the spatial components of the perturbed EMT

$$
\begin{aligned}
T^{i j}-T_{0}^{i j}= & \left\langle\frac{p^{i} p^{j}}{p^{0}} \mid \phi\right\rangle, \\
= & \frac{1}{3} \delta^{i j}\left\{k^{2} h_{S} \frac{T_{0}^{4} \Gamma[5]}{6 \pi^{2}}\left(1+\frac{k^{2}}{3 \omega^{2}}\right)-\frac{k_{k} k_{l}}{\omega^{2}} \sum_{\sigma}\left\langle\frac{p^{k} p^{l}}{p^{0}} \mid \phi_{\sigma}\right\rangle \frac{\omega h_{l m}}{2 T_{0}\left[\omega+i \nu_{\sigma}\right]}\left\langle\phi_{\sigma} \mid \frac{p^{l} p^{m}}{p^{0}}\right\rangle\right\} \\
& +\sum_{\lambda}\left\langle\frac{p^{i} p^{j}}{p^{0}} \mid \phi_{\lambda}\right\rangle\left\{-\frac{\omega h_{l m}}{2 T_{0}\left[\omega+i \nu_{\lambda}\right]}\left\langle\phi_{\lambda} \mid \frac{p^{l} p^{m}}{p^{0}}\right\rangle\right. \\
& +\frac{k_{k}}{\left(\omega+i \nu_{\lambda}\right)}\left\{\sum_{l} 3 \alpha_{0}\left\langle\phi_{\lambda} \mid \frac{p^{k} p^{l}}{p^{0}}\right\rangle \frac{1}{\omega}\left\{k^{l} k^{2} h_{S} \frac{T_{0}^{4} \Gamma[5]}{18 \pi^{2}}-\sum_{\sigma} k_{m}\left\langle\frac{p^{l} p^{m}}{p^{0}} \mid \phi_{\sigma}\right\rangle \frac{\omega h_{p q}}{2 T_{0}\left[\omega+i \nu_{\sigma}\right]}\left\langle\phi_{\sigma} \mid \frac{p^{p} p^{q}}{p^{0}}\right\rangle\right\}\right. \\
& \left.\left.-\sum_{\sigma}\left\langle\phi_{\lambda}\left|\hat{p}^{k}\right| \phi_{\sigma}\right\rangle\left\{\frac{k_{l}}{\left(\omega+i \nu_{\sigma}\right)} \sum_{\sigma^{\prime}}\left\langle\phi_{\sigma}\left|\hat{p}^{l}\right| \phi_{\sigma^{\prime}}\right\rangle \frac{\omega h_{p q}}{2 T_{0}\left[\omega+i \nu_{\sigma^{\prime}}\right]}\left\langle\phi_{\sigma^{\prime}} \mid \frac{p^{p} p^{q}}{p^{0}}\right\rangle\right\}\right\}\right\} .
\end{aligned}
$$

We observe that there must be eigenvectors for which $\left\langle\left(p^{i} p^{j} / p^{0}\right) \mid \phi_{\lambda}\right\rangle \neq 0$, since $\left|\left(p^{i} p^{j} / p^{0}\right)\right\rangle$ is not a linear superposition of the null eigenvectors $\left|p^{\mu}\right\rangle$ and the $\left|\phi_{\lambda}\right\rangle$ are complete in the complement of the $\left|p^{\mu}\right\rangle$. In coordinates where $k^{i}$ is in the $z$ direction, this is true in particular for $\left|p^{x} p^{y} / p^{0}\right\rangle$, which couples to the tensor part of $h_{i j},\left|p^{x} p^{z}\right|$ $\left.p^{0}\right\rangle$, which couples to the vector part, and $\mid\left(p^{x 2}+p^{y 2}-\right.$ $\left.\left.2 p^{z 2}\right) / p^{0}\right\rangle$, which couples to the scalar part. So there must be a nontrivial propagator for tensor modes just as there is for vector and scalar ones. Also, if the collision term does not break the isotropy of space, we expect the eigenvectors to carry a representation of the rotation group, and for this reason at least, the eigenvectors that couple to these kets (and therefore cannot be rotation invariant) will be degenerate.

Once we have the representation (74) at our disposal, it is a simple matter to match it to the asympotic behavior resulting from a suitable distribution of poles and cuts in the complex frequency plane. For example, in the first term of Eq. (74), we find

$$
\left(1+\frac{k^{2}}{3 \omega^{2}}\right) \approx \frac{\omega^{2}}{\omega^{2}-\left(k^{2} / 3\right)}
$$

signaling the presence of the usual sound pole. Then it is easy to see that eigenvalues in the discrete spectrum will give rise to poles, while eigenvalues in the continuous spectrum will be associated to cuts. This derives from the fact that an expression such as

$$
\int_{\nu_{0}}^{\infty} d \nu \frac{\rho[\nu]}{[\omega+i \nu]}
$$

is discontinuous when we go from $\omega=-i \nu+\epsilon$ to $\omega=-i \nu-\epsilon$, for all $\nu>\nu_{0}$ such that $\rho[\nu] \neq 0$.

The relaxation time approximation is the extreme case where the full spectrum is collapsed to just two points, 0 and $1 / \tau$. The sums over the non-null eigenvalues are performed by using $\sum_{\sigma}\left\langle\psi^{\prime} \mid \phi_{\sigma}\right\rangle\left\langle\phi_{\sigma} \mid \psi\right\rangle=\left\langle\psi^{\prime} \mid \psi\right\rangle-\sum_{\nu=0}^{3} \alpha_{\nu}\left\langle\psi^{\prime} \mid p^{\nu}\right\rangle\left\langle p^{\nu} \mid \psi\right\rangle$,

and we easily recover the results above. In the opposite limit, the theories with an energy dependent relaxation time discussed in [22] (see also [105-108]) have purely continuous spectrum ranging from $\nu_{0}=0$ to $\infty$, and therefore lead to an analytic structure dominated by cuts.

Realistic kinetic equations such as Boltzmann's [109-111] and Landau's [112,113] have both isolated and continuous eigenvalues, strongly dependent upon the details of the interparticle interactions. The so-called hard potentials have a continuous spectrum ranging from a finite value $\nu_{0}>0$ to $\infty$ [114]; they may have further isolated eigenvalues between the ever-present 0 and $\nu_{0}[115,116]$, and besides, since most modes in the continuum decay much faster than $\nu_{0}^{-1}$, it may be possible, for all practical purposes, to approximate the continuum by a single eigenvalue. Contrariwise, "soft" interactions lead to a continuous spectrum starting from $\nu_{0}=0$ [114]. We should note that both Boltzmann's and Landau's equations have a fifth null eigenvector associated with particle number conservation, which is not a feature of conformal theories (in gauge theories there are particle number changing processes such as gluon splitting, in $\lambda \phi^{4}$ theory particle number conservation is broken at order $\lambda^{4}$ [117]). More general collision terms are discussed in [3].

We therefore conclude that the analytic structure found under the relaxation time approximation is not an artifact of the approximation, although it is not universal either, and its validity must be judged on a case by case basis.

\section{DISPERSION RELATIONS IN DIVERGENCE TYPE THEORIES}

In this section we shall compute the dispersion relations as derived from SOTs within a DTT scheme. 
Let us motivate this particular SOT by deriving it from kinetic theory [15]. To this end we assume a parametrized distribution function

$$
f=f_{\text {hydro }}\left[x^{\mu}, p_{\mu} ; \beta^{\mu}, \zeta^{\mu \nu}, \ldots\right] .
$$

We obtain equations of motion for the parameters $\beta^{\mu}, \zeta^{\mu \nu}, \ldots$ by taking suitable averages of the Boltzmann equation $[74,75]$

$$
\begin{aligned}
\int D p p^{\mu} p^{\nu} f_{\mathrm{hydr} ; \nu} & =\int D p p^{\mu} I_{\mathrm{col}}\left[f_{\mathrm{hydro}}\right]=0, \\
\int D p R_{\alpha}[x, p] p^{\nu} f_{\mathrm{hydro} ; \nu} & =\int D p R_{\alpha} I_{\mathrm{col}}\left[f_{\mathrm{hydro}}\right] \ldots
\end{aligned}
$$

This scheme enforces energy-momentum conservation [Eq. (1)]. However, because $f_{\text {hydro }}$ is not a solution of the Boltzmann equation (14), the $H$ theorem (20) does not apply to it, and the Second Law may not be enforced. This is avoided if we make the specific parametrization

$$
f_{\text {hydro }}=e^{\beta^{\mu} p_{\mu}+\sum_{\alpha} \zeta^{\alpha} R_{\alpha}[x, p]},
$$

that is, the same functions $R_{\alpha}$ which appear in $f_{\text {hydro }}$ are averaged against the Boltzmann equation to obtain the equations of motion for the parameters $\zeta^{\alpha}$ [15]. The resulting system of equations take the form of conservation laws for the currents

$$
A_{\alpha}^{\mu}=\int D p R_{\alpha}[x, p] p^{\mu} f_{\text {hydro }}
$$

and so this theory falls within the class of DTTs.

The particular implementation of this scheme we are interested in postulates two nonequilibrium tensors $\zeta_{\mu \nu}$ and $\xi_{\mu \nu \rho}$ besides the usual variables $T$ and $u^{\mu}$. The parametrized distribution function reads

$$
f_{\text {hydro }}=e^{\beta_{\mu} p^{\mu}+\zeta_{\mu \nu} \frac{p^{\mu} p^{\nu}}{\left(-u_{\lambda} p^{\lambda}\right)}+\xi_{\mu \nu \rho} \frac{p^{\mu} \nu^{\nu} p^{\rho}}{\left(-u_{\lambda} p^{\lambda}\right)^{2}}}
$$

where $\beta^{\mu}=u^{\mu} / T, u^{2}=-1$. $\zeta_{\mu \nu}$ and $\xi_{\mu \nu \rho}$ are totally symmetric, traceless and transverse, meaning that

$$
\begin{aligned}
S_{\sigma \lambda}^{\mu \nu} \zeta^{\sigma \lambda} & =\zeta^{\mu \nu}, \\
S_{\sigma \lambda \tau}^{\mu \nu \rho} \xi \sigma \lambda \tau & =\xi^{\mu \nu \rho},
\end{aligned}
$$

with the projectors

$$
\begin{aligned}
S_{\sigma \lambda}^{\mu \nu}= & \frac{1}{2}\left\{\Delta_{\sigma}^{\mu} \Delta_{\lambda}^{\nu}+\Delta_{\lambda}^{\mu} \Delta_{\sigma}^{\nu}-\frac{2}{3} \Delta^{\mu \nu} \Delta_{\sigma \lambda}\right\}, \\
S_{\sigma \lambda \tau}^{\mu \nu \rho}= & \frac{1}{6}\left\{\Delta_{\sigma}^{\mu} \Delta_{\lambda}^{\nu} \Delta_{\tau}^{\rho}+\Delta_{\sigma}^{\mu} \Delta_{\tau}^{\nu} \Delta_{\lambda}^{\rho}+\Delta_{\lambda}^{\mu} \Delta_{\sigma}^{\nu} \Delta_{\tau}^{\rho}\right. \\
& +\Delta_{\lambda}^{\mu} \Delta_{\tau}^{\nu} \Delta_{\sigma}^{\rho}+\Delta_{\tau}^{\mu} \Delta_{\lambda}^{\nu} \Delta_{\sigma}^{\rho}+\Delta_{\tau}^{\mu} \Delta_{\sigma}^{\nu} \Delta_{\lambda}^{\rho} \\
& -\frac{2}{5}\left[\Delta^{\mu \nu}\left(\Delta_{\sigma}^{\rho} \Delta_{\lambda \tau}+\Delta_{\lambda}^{\rho} \Delta_{\sigma \tau}+\Delta_{\tau}^{\rho} \Delta_{\lambda \sigma}\right)\right. \\
& +\Delta^{\mu \rho}\left(\Delta_{\sigma}^{\nu} \Delta_{\lambda \tau}+\Delta_{\lambda}^{\nu} \Delta_{\sigma \tau}+\Delta_{\tau}^{\nu} \Delta_{\lambda \sigma}\right) \\
& \left.\left.+\Delta^{\nu \rho}\left(\Delta_{\sigma}^{\mu} \Delta_{\lambda \tau}+\Delta_{\lambda}^{\mu} \Delta_{\sigma \tau}+\Delta_{\tau}^{\mu} \Delta_{\lambda \sigma}\right)\right]\right\},
\end{aligned}
$$

where as usual $\Delta^{\mu \nu}=g^{\mu \nu}+u^{\mu} u^{\nu}$.

We should note that most work on DTTs to date does not include the third order tensor $\xi_{\mu \nu \rho}$. Often the only variables considered are a chemical potential (for nonconformal theories), the four vector $\beta_{\mu}$ and a traceless tensor $\zeta_{\mu \nu}$ [118]. This adds up to 14 degrees of freedom, and is thus analog to Grad's "14 moments" approximation. In these usual theories the tensor mode in nonpropagating, while, as we shall show below, including the $\xi_{\mu \nu \rho}$ tensor provides it with a finite propagation speed, which further agrees with the one derived from kinetic theory (55).

Including the tensor $\xi_{\mu \nu \rho}$ will not just produce this only change in the theory, we should expect there will be incremental changes in the scalar and vector sectors as well. However it is fair to say that they do not change the physical picture in those sectors as they do for the tensor modes. Likewise, including higher order tensors will only have incremental effects on $T^{\mu \nu}$.

The equations of motion for the variables $\beta^{\mu}, \zeta^{\mu \nu}$ and $\xi^{\mu \nu \rho}$ are obtained from the weighted averages of the covariant Boltzmann equation (14)

$$
\begin{array}{r}
\int D p p^{\mu}\left[p^{\tau} f_{\mathrm{hydro} ; \tau}-I_{\mathrm{col}}\right]=0, \\
\int D p S_{\mu \nu}^{\alpha \beta} \frac{p^{\mu} p^{\nu}}{\left(-u_{\lambda} p^{\lambda}\right)}\left[p^{\tau} f_{\mathrm{hydro} ; \tau}-I_{\mathrm{col}}\right]=0, \\
\int D p S_{\mu \nu \rho}^{\alpha \beta \gamma} \frac{p^{\mu} p^{\nu} p^{\rho}}{\left(-u_{\lambda} p^{\lambda}\right)^{2}}\left[p^{\tau} f_{\mathrm{hydro} ; \tau}-I_{\mathrm{col}}\right]=0 .
\end{array}
$$

Integrating by parts, we write these equations as conservation laws

$$
\begin{aligned}
T_{; \nu}^{\mu \nu} & =0, \\
S_{\mu \nu}^{\alpha \beta}\left[A_{; \rho}^{\mu \nu \rho}-A^{\mu \nu \rho \sigma} u_{\rho ; \sigma}-I^{\mu \nu}\right] & =0, \\
S_{\mu \nu \rho}^{\alpha \beta \gamma}\left[A_{; \sigma}^{\mu \nu \rho \sigma}-2 A^{\mu \nu \rho \sigma \lambda} u_{\sigma ; \lambda}-I^{\mu \nu \rho}\right] & =0,
\end{aligned}
$$

where 


$$
\begin{aligned}
T^{\mu \nu} & =\int D p p^{\mu} p^{\nu} f_{\text {hydro }}, \\
A^{\mu_{1} \ldots \mu_{n}} & =\int D p \frac{p^{\mu_{1}} \ldots p^{\mu_{n}}}{\left(-u_{\lambda} p^{\lambda}\right)^{n-2}} f_{\text {hydro }}, \\
I^{\mu_{1} \ldots \mu_{n}} & =\int D p \frac{p^{\mu_{1}} \ldots p^{\mu_{n}}}{\left(-u_{\lambda} p^{\lambda}\right)^{n-1}} I_{\mathrm{col}} .
\end{aligned}
$$

If $I_{\text {col }}$ satisfies the $H$ theorem, then this dynamics yields positive entropy production, with the entropy flux

$$
\begin{aligned}
S^{\mu} & =\int D p p^{\mu} f_{\text {hydro }}\left[1-\ln f_{\text {hydro }}\right], \\
& =\Phi^{\mu}-\beta_{\nu} T^{\mu \nu}-\zeta_{\nu \rho} A^{\mu \nu \rho}-\xi_{\nu \rho \sigma} A^{\mu \nu \rho \sigma},
\end{aligned}
$$

and entropy production

$$
\Sigma=-\zeta_{\nu \rho} I^{\nu \rho}-\xi_{\nu \rho \sigma} I^{\nu \rho \sigma}
$$

Here

$$
\Phi^{\mu}=\int D p p^{\mu} f_{\text {hydro }}
$$

So far the discussion has been general. We now linearize around an equilibrium solution. We adopt the relaxation time approximation collision integral (22).

In equilibrium $\zeta^{\mu \nu}=\xi^{\mu \nu \rho}=0$, since we are interested in linear deviations from equilibrium only, we can write

$$
f_{\text {hydro }}=e^{\beta_{\mu} p^{\nu}}\left[1+\zeta_{\mu \nu} \frac{p^{\mu} p^{\nu}}{\left(-u_{\lambda} p^{\lambda}\right)}+\xi_{\mu \nu \rho} \frac{p^{\mu} p^{\nu} p^{\rho}}{\left(-u_{\lambda} p^{\lambda}\right)^{2}}\right]
$$

then also $f_{e q}=e^{\beta_{\mu} p^{\nu}}$ and the equations of motion reduce to

$$
\begin{aligned}
T_{0 ; \nu}^{\mu \nu}+T_{1}^{\mu \nu \rho \sigma} \zeta_{\rho \sigma ; \nu} & =0, \\
S_{\mu \nu}^{\alpha \beta}\left[T_{1 ; \rho}^{\mu \nu \rho}+T_{1}^{\mu \nu \rho \sigma}\left(\dot{\zeta}_{\rho \sigma}+\frac{1}{\tau} \zeta_{\rho \sigma}\right)+T_{3}^{\mu \nu \rho \sigma \lambda \tau} \xi_{\sigma \lambda \tau ; \rho}-T_{2}^{\mu \nu \rho \sigma} u_{\rho ; \sigma}\right] & =0, \\
S_{\mu \nu \rho}^{\alpha \beta \gamma} T_{3}^{\mu \nu \rho \sigma \lambda \tau}\left[\dot{\xi}_{\sigma \lambda \tau}+\frac{1}{\tau} \xi_{\sigma \lambda \tau}+\zeta_{\lambda \sigma ; \tau}\right] & =0,
\end{aligned}
$$

where

$$
T_{\alpha}^{\mu_{1} \ldots \mu_{n}}=\int D p \frac{p^{\mu_{1}} \ldots p^{\mu_{n}}}{\left(-u_{\lambda} p^{\lambda}\right)^{\alpha}} f_{\mathrm{eq}} .
$$

These tensors are evaluated in Appendix C. In general, the projectors mean that we have to symmetrize and subtract all longitudinal and tracefull terms. We also write $T=T_{0}(1+\vartheta)$ and $u^{\mu}=U^{\mu}+v^{\mu}$, with $U^{\mu} v_{\mu}=0$. We then get

$$
\begin{aligned}
\dot{\vartheta}+\frac{1}{3}\left(v_{, i}^{i}+\frac{1}{2} \dot{h}_{i}^{i}\right) & =0, \\
\dot{v}_{j}+\vartheta_{, j}+\frac{2}{5} T_{0} \zeta_{j, k}^{k} & =0, \\
\frac{1}{2} \sigma_{j k}+T_{0}\left(\dot{\zeta}_{j k}+\frac{1}{\tau} \zeta_{j k}\right)+\frac{3}{7} T_{0} \xi_{j k l, l} & =0, \\
\frac{1}{3}\left[\zeta_{i j, k}+\zeta_{i k, j}+\zeta_{j k, i}-\frac{2}{5}\left(\delta_{i j} \zeta_{k l, l}+\delta_{i k} \zeta_{j l, l}+\delta_{k j} \zeta_{i l, l}\right)\right]+\dot{\xi}_{i j k}+\frac{1}{\tau} \xi_{i j k} & =0,
\end{aligned}
$$

where $\sigma_{i j}$ is the shear tensor

$$
\sigma_{j k}=v_{i, j}+v_{j, i}-\frac{2}{3} \delta_{i j} v_{, k}^{k}+\dot{h}_{i j}-\frac{1}{3} \delta_{i j} \dot{h}_{k}^{k}
$$

Fourier transforming and using the velocity decomposition (27) and the metric decomposition (28) we get

$$
\sigma_{j k}=-2\left[k_{i} k_{j}-\frac{1}{3} \delta_{i j} k^{2}\right] v_{S}+i\left(k_{i} v_{V j}+k_{j} v_{V i}\right)-i \omega\left[\left[k_{i} k_{j}-\frac{1}{3} \delta_{i j} k^{2}\right]\left(h_{S}+h_{S}^{\prime}\right)+i k_{i} h_{V j}+i k_{j} h_{V i}+h_{T i j}\right] .
$$


To decompose Eq. (94) in scalar, vector and tensor modes, we define

$$
\begin{aligned}
\zeta_{i j}= & \zeta_{S}\left[k_{i} k_{j}-\frac{1}{3} k^{2} \delta_{i j}\right]+i\left(\zeta_{V i} k_{j}+\zeta_{V j} k_{i}\right)+\zeta_{T i j}, \\
\xi_{i j k}= & i \xi_{S}\left[k_{i} k_{j} k_{k}-\frac{1}{5} k^{2}\left(k_{i} \delta_{j k}+k_{j} \delta_{i k}+k_{k} \delta_{i j}\right)\right] \\
& +\xi_{V i}\left[k_{j} k_{k}-k^{2} \frac{1}{5} \delta_{j k}\right]+\xi_{V j}\left[k_{i} k_{k}-k^{2} \frac{1}{5} \delta_{i k}\right] \\
& +\xi_{V k}\left[k_{j} k_{i}-k^{2} \frac{1}{5} \delta_{j i}\right]+i\left(\xi_{T i j} k_{k}+\xi_{T i k} k_{j}+\xi_{T k j} k_{i}\right) \\
& +\xi_{T T i j k},
\end{aligned}
$$

where $k_{i} \zeta_{V i}=k_{i} \zeta_{T i j}=0, \quad k_{i} \xi_{V i}=k_{i} \xi_{T i j}=k_{i} \xi_{T T i j k}=0$, and tensors are totally symmetric and traceless. The scalar-vector-tensor decomposition of the shear tensor is given in Eq. (96).

\section{A. The scalar sector}

In the scalar sector we get

$$
\begin{aligned}
i \omega \vartheta+\frac{1}{3} k^{2} v_{S} & =\frac{i}{3} \omega k^{2} h_{S}, \\
\vartheta-i \omega v_{S}+\frac{4}{15} k^{2} T_{0} \zeta_{S} & =0, \\
v_{S}+i\left(\omega+\frac{i}{\tau}\right) T_{0} \zeta_{S}+\frac{9}{35} k^{2} T_{0} \xi_{S} & =\frac{1}{2} i \omega\left(h_{S}+h_{S}^{\prime}\right), \\
\zeta_{S}-i\left(\omega+\frac{i}{\tau}\right) \xi_{S} & =0 .
\end{aligned}
$$

The dispersion relation is

$$
\begin{aligned}
& \left(\omega^{2}-\frac{1}{3} k^{2}\right)\left(\omega+\frac{i}{\tau}\right)^{2} \\
& \quad-k^{2}\left[\frac{4}{15} \omega\left(\omega+\frac{i}{\tau}\right)+\frac{9}{35}\left(\omega^{2}-\frac{1}{3} k^{2}\right)\right]=0 .
\end{aligned}
$$

When $k^{2}, \tau \rightarrow 0$ we have two branches of solutions, hydrodynamic modes with

$$
\omega \approx \pm \frac{k}{\sqrt{3}}-\frac{2}{15} i \tau k^{2},
$$

which correspond to the Landau-Lifshitz modes with the identification $\nu=\tau / 5$ (see Appendix A), and nonhydrodynamic modes with

$$
\omega \approx \frac{-i}{\tau} \pm \sqrt{\frac{9}{35}} k
$$

just as derived from kinetic theory, Eq. (49).

\section{B. The vector sector}

In the vector sector we get

$$
\begin{aligned}
\omega v_{V j}-\frac{2}{5} i k^{2} T_{0} \zeta_{V j} & =0 \\
\frac{1}{2} i v_{V j}+\left(\omega+\frac{i}{\tau}\right) T_{0} \zeta_{V j}+\frac{12}{35} i k^{2} T_{0} \xi_{V j} & =-\frac{1}{2} i \omega h_{V j}, \\
\frac{2}{3} i \zeta_{V j}-\left(\omega+\frac{i}{\tau}\right) \xi_{V j} & =0 .
\end{aligned}
$$

The dispersion relation is

$$
\omega\left(\omega+\frac{i}{\tau}\right)^{2}-\frac{1}{5} k^{2}\left(\omega+\frac{i}{\tau}\right)-\frac{8}{35} k^{2} \omega=0 .
$$

Therefore when $k^{2} \rightarrow 0$, either $\omega \rightarrow 0$ or $\omega \rightarrow-i \tau^{-1}$. In the first case we find an hydrodynamic mode with

$$
\omega \approx-\frac{1}{5} i \tau k^{2}
$$

while the others are two nonhydrodynamic modes with

$$
\omega \approx \frac{-i}{\tau} \pm \sqrt{\frac{8}{35}} k
$$

Once again, the hydrodynamic modes agree with LandauLifshitz theory if $\nu=\tau / 5$ (see Appendix A), and also reproduces the nonhydrodynamic mode from kinetic theory (52).

\section{The tensor sector}

In the tensor sector we get

$$
\begin{aligned}
\left(\omega+\frac{i}{\tau}\right) \zeta_{T j k}-\frac{3}{7} i k^{2} \xi_{T j k} & =\frac{-i}{T_{0}} \omega h_{T j k}, \\
\frac{1}{3} i \zeta_{T j k}+\left(\omega+\frac{i}{\tau}\right) \xi_{T j k} & =0 .
\end{aligned}
$$

We therefore find two nontrivial hydrodynamic modes with

$$
\omega \approx \frac{-i}{\tau} \pm \sqrt{\frac{1}{7}} k
$$

These modes have no analog in Landau-Lifshitz fluids, but match quantitatively the nonhydrodinamic tensor modes from kinetic theory (55).

If we had considered a truncated theory with $\xi_{i j k}=0$, then there would be a tensor nonhydrodynamic mode, but with a $k$-independent dispersion relation $\omega=-i / \tau$, it is thus not propagating. They are present already in the IsraelStewart theory $[119,120]$. Their cosmological consequences are discussed in $[121,122]$. 
$\xi_{T T i j k}$ is decoupled and obeys the equation

$$
\left(\omega+\frac{i}{\tau}\right) \xi_{T T i j k}=0
$$

so we may assume it vanishes throughout.

\section{FINAL REMARKS}

In this paper we have computed the dispersion relations for a DTT containing two nonequilibrium tensors $\zeta_{\mu \nu}$ and $\xi_{\mu \nu \rho}$ besides the usual variables $T$ and $u^{\mu}$, and compared the result to known results in the literature involving FOTs [14] and also "first principles" calculations from kinetic theory [21-24] and quantum field theory [1,20,25-35].

The "first principles" calculations display both hydrodynamic and nonhydrodynamic modes in all three sectors, scalar vector and tensor. Both FOTs and DTTs describe well the hydrodynamic modes and the longest living nonhydrodynamic modes in the scalar and vector sectors. DTTs also describe the longest living tensor mode, which is not recovered in FOTs. A truncated DTT with only the $\zeta_{\mu \nu}$ tensor yields a nonpropagating tensor mode, in agreement with Israel-Stewart theory $[119,120]$.

We have been unable to find a clear cut statement about the speed of propagation of tensor modes in the third order formalism $[59,61,123,124]$, since usually only the "sound" and "shear" channels (which correspond to scalar and vector modes in this paper) are discussed in detail. The relevant third order equation as derived in $[60,125]$ includes second order derivatives of the viscous EMT.

DTTs also perform well in the similar problem of the dispersion relations for a viscous charged fluid [126,127], where they reproduce the Weibel instability $[128,129]$. The cosmological consequences of this fact are discussed in [130].

We believe these results validate the choice of DTTs to describe fluids in problems where the interaction with gravitational waves is a matter of relevance [131]. These problems include generation and amplification of gravitational waves in the very early Universe $[121,122,132]$ and during cosmological phase transitions [133], and gravitational wave emission from rotating neutron stars [134], among others. Where gravitational interactions are not a concern, the relative advantages of FOTs and SOTs must be considered carefully to find the most suitable model for each application.

\section{ACKNOWLEDGMENTS}

We thank M. Nigro, A. Kandus, N. Mirón Granese, L. Cantarutti and Julián Ruffinelli for multiple discussions. This work was supported in part by Universidad de Buenos Aires (Grant UBACYT No. 20020170100129BA); CONICET (Grant PIP 11220170100817CO); ANPCYT (Grant PICT 03684).

\section{APPENDIX A: DISPERSION RELATIONS FOR IDEAL AND LANDAU-LIFSHITZ FLUIDS, AND IN CAUSAL FOTS}

\section{Ideal fluids}

To obtain the dispersion relations for an ideal fluid we must consider the conservation laws (1) for the EMT (3). For a conformal fluid, $\rho=\sigma T^{4}$ and $p=\rho / 3$. For concreteness we assume the value of the constant $\sigma$ which is compatible with Maxwell-Jüttner statistics for a single degree of freedom, namely $\sigma=3 / \pi^{2}$. The conservation laws are

$$
\begin{aligned}
\frac{\dot{T}}{T}+\frac{1}{3} u_{; \nu}^{\nu} & =0, \\
\dot{u}^{\mu}+\Delta^{\mu \nu} \frac{T_{, \nu}}{T} & =0,
\end{aligned}
$$

$\dot{X}=u^{\mu} X_{; \mu}$. We linearize these equations by writing $T=T_{0}(1+\vartheta), \quad u^{\mu}=U^{\mu}+v^{\mu}, \quad U^{\mu}=(1,0,0,0), \quad$ and $v^{0}=0$. Taking the Fourier transform we get

$$
\begin{aligned}
-i \omega \vartheta+\frac{1}{3}\left[i k_{j} v^{j}-\frac{i}{2} \omega h_{i}^{i}\right] & =0, \\
-i \omega v^{j}+i k_{j} \vartheta & =0 .
\end{aligned}
$$

We now decompose the velocity as in Eq. (27) and the metric as in Eq. (28). No tensor degrees of freedom are included. We thereby get on one hand

$$
\begin{aligned}
-\omega \vartheta+\frac{i}{3} k^{2} v_{S} & =-\frac{1}{3} \omega k^{2} h_{S}, \\
\vartheta-i \omega v_{S} & =0,
\end{aligned}
$$

and on the other

$$
\omega v_{V}^{j}=0 .
$$

We see that scalar, vector and tensor modes decouple. For the scalar modes we find the dispersion relation

$$
\omega^{2}-\frac{1}{3} k^{2}=0,
$$

so the scalar propagator has poles at $\omega= \pm k / \sqrt{3}$, representing longitudinal sound waves. No vector or tensor modes are excited in the fluid.

\section{Landau-Lifshitz fluids}

If we consider a Landau-Lifshitz fluid instead of an ideal one, the difference is that the EMT becomes

$$
T^{\mu \nu}=\frac{4}{3} \sigma T^{4}\left[u^{\mu} u^{\nu}+\frac{1}{4} g^{\mu \nu}\right]-\eta \sigma^{\mu \nu},
$$


where $\eta$ is the fluid viscosity and $\sigma^{\mu \nu}$ is the shear tensor

$$
\sigma^{\mu \nu}=\Delta^{\mu \lambda} \Delta^{\nu \rho}\left[u_{\lambda ; \rho}+u_{\rho ; \lambda}-\frac{2}{3} \Delta_{\lambda \rho} u_{; \sigma}^{\sigma}\right] .
$$

The linearized conservation equations are now

$$
\begin{aligned}
\dot{\vartheta}+\frac{1}{3} v_{; j}^{j} & =0, \\
\dot{v}^{j}+\vartheta_{, j}-\nu \sigma_{; k}^{j k} & =0,
\end{aligned}
$$

where $\nu=3 \eta / 4 \sigma T_{0}^{4}=\eta / s_{0} T_{0}$ is the kinematic viscosity. With the shear tensor (95) and its Fourier transform (96) we get

$$
\begin{gathered}
-\omega \vartheta+\frac{i}{3} k^{2} v_{S}=-\frac{1}{3} \omega k^{2} h_{S}, \\
\vartheta-i\left[\omega+\frac{4 i}{3} \nu k^{2}\right] v_{S}=\frac{1}{2} i k^{2} \nu \omega\left(h_{S}+h_{S}^{\prime}\right),
\end{gathered}
$$

and

$$
\left[\omega+i \nu k^{2}\right] v_{V}^{j}=-\frac{3}{4} \nu k^{2} \omega h_{V}^{j}
$$

We see that the longitudinal sound waves now become damped, with a dispersion relation

$$
\omega \approx \pm \frac{k}{\sqrt{3}}-\frac{2}{3} i \nu k^{2}
$$

while the pole for transverse waves has been shifted from $\omega=0$ to $\omega=-i \nu k^{2}$. There are still no tensor modes in the fluid.

\section{Causal FOTs}

As a representative causal FOT we shall consider the EMT [14]

$$
\begin{aligned}
T^{\mu \nu}= & {\left[\epsilon+\frac{3 \chi}{4 \epsilon}\left(u^{\rho} \nabla_{\rho} \epsilon+\frac{4}{3} \epsilon \nabla_{\rho} u^{\rho}\right)\right]\left(u^{\mu} u^{\nu}+\frac{1}{3} \Delta^{\mu \nu}\right)-\eta \sigma^{\mu \nu} } \\
& +\frac{\lambda}{4 \epsilon}\left[4 u^{\mu} \epsilon u^{\rho} \nabla_{\rho} u^{\nu}+u^{\mu} \Delta_{\rho}^{\nu} \nabla^{\rho} \epsilon+4 u^{\nu} \epsilon u^{\rho} \nabla_{\rho} u^{\mu}+u^{\nu} \Delta_{\rho}^{\mu} \nabla^{\rho} \epsilon\right]
\end{aligned}
$$

Only when in equilibrium, $\epsilon=\rho$ is the energy density. The new transport coeffcients $\chi$ and $\lambda$ are the hallmark of the causal FOT approach. They define timescales of the order of the relaxation time on which the hydrodynamic variable $\epsilon$ relaxes to the Landau-Lifshitz energy density, and in this sense they act as causal regulators. See [14] for further discussion.

To linearize the conservation equations (1) we write $\epsilon=\epsilon_{0}+\delta \epsilon$, and expand $u^{\mu}=U^{\mu}+v^{\mu}$ and $g^{\mu \nu}=\eta^{\mu \nu}+h^{\mu \nu}$ as before. We further decompose $v^{i}$ as in Eq. (27) and $h_{i j}$ as in Eq. (28). We thus get the Fourier-transformed equations

$$
\begin{aligned}
0 & =-i \omega\left[\delta \epsilon+\frac{3 \chi}{4 \epsilon_{0}}\left(-i \omega \delta \epsilon-\frac{4}{3} \epsilon_{0} k^{2} v_{S}\right)\right]-\left[\frac{4}{3} \epsilon_{0}-\lambda i \omega\right] k^{2} v_{S}-\frac{1}{6} \epsilon_{0} i \omega h_{S}, \\
0= & -i \omega\left[\frac{4}{3} \epsilon_{0}-\lambda i \omega\right]\left(i k^{i} v_{S}+v_{V}^{i}\right)+\frac{1}{3} \epsilon_{0}\left(-i \frac{2}{3} k^{2} k^{i} h_{S}^{\prime}-k^{2} h_{V}^{i}\right)+\frac{1}{6} i \epsilon_{0} k^{i} k^{2} h_{S} \\
& +\frac{1}{3} i k^{i}\left[\delta \epsilon+\frac{3 \chi}{4 \epsilon_{0}}\left(-i \omega \delta \epsilon-\frac{4}{3} \epsilon_{0} k^{2} v_{S}\right)\right]-\frac{1}{3} \epsilon_{0}\left[\frac{2}{3} i k^{2} k^{i} h_{S}^{\prime}+k^{2} h_{V}^{i}\right] \\
& +2 \eta\left[\frac{1}{2}\left(k^{2}\left(i k^{i} v_{S}+v_{V}^{i}\right)+i k^{i}\left(k^{2} v_{S}\right)-i \omega k^{2} i k^{i} h_{S}^{\prime}\right)-\frac{1}{3} i k^{i}\left(-k^{2} v_{S}+i \omega k^{2} h_{S}\right)\right] .
\end{aligned}
$$

We already see that the tensor sector has trivial dynamics. We analyze the scalar and vector sectors in turn.

\section{a. Scalar sector}

The scalar part of Eq. (A14) reads

$$
\begin{aligned}
0= & -i \omega\left[\frac{4}{3} \epsilon_{0}-\lambda i \omega\right] v_{S}-\frac{4}{9} \epsilon_{0} k^{2} h_{S}^{\prime}+\frac{1}{6} \epsilon_{0} k^{2} h_{S}+\frac{1}{3}\left[\delta \epsilon+\frac{3 \chi}{4 \epsilon_{0}}\left(-i \omega \delta \epsilon-\frac{4}{3} \epsilon_{0} k^{2} v_{S}\right)\right] \\
& +2 \eta k^{2}\left[\frac{2}{3} v_{S}-\frac{1}{2} i \omega h_{S}^{\prime}+\frac{1}{3} i \omega h_{S}\right] .
\end{aligned}
$$


Elliminating $v_{S}$ we find

$$
\begin{gathered}
\frac{\delta \epsilon}{\epsilon_{0}}\left[1-\frac{\epsilon_{0} k^{2}}{3 i \omega} \frac{\left[\frac{4}{3}-i \omega\left(\frac{\lambda+\chi}{\epsilon_{0}}\right)\right]}{\left[-\omega^{2} \lambda-i \omega \frac{4}{3} \epsilon_{0}-\frac{1}{3} k^{2}(\chi-4 \eta)\right]}\right]=-\frac{1}{6} \frac{1}{\left[1-i \omega \frac{3 \chi}{4 \epsilon_{0}}\right]} h_{S} \\
+\frac{k^{2}}{i \omega} \frac{\left[\frac{4}{3}-i \omega\left(\frac{\lambda+\chi}{\epsilon_{0}}\right)\right]}{1-i \omega \frac{3 \chi}{4 \epsilon_{0}}}\left\{\frac{\frac{2}{3} \epsilon_{0} k^{2}\left(\frac{2}{3} h_{S}^{\prime}-\frac{1}{4} h_{S}\right)-\eta i \omega k^{2}\left(\frac{2}{3} h_{S}-h_{S}^{\prime}\right)}{-\omega^{2} \lambda-i \omega \frac{4}{3} \epsilon_{0}+\frac{1}{3} k^{2}(\chi-4 \eta)}\right\} .
\end{gathered}
$$

In the limit $k^{2} \rightarrow 0 \mathrm{Eq}$. (A16) simplifies to

$$
\frac{\delta \epsilon}{\epsilon_{0}}=-\frac{1}{\left[1-i \omega \frac{3 \chi}{4 \epsilon_{0}}\right]} \frac{1}{\left[\omega^{2}-\frac{1}{3} k^{2}(1-\alpha)\right]} \frac{h_{S}}{6}
$$

where

$$
\alpha=\frac{3}{4} i \omega \frac{\chi}{\epsilon_{0}}\left[1-\frac{3}{4} i \omega \frac{\lambda}{\epsilon_{0}}\right]^{-1}
$$

We therefore get a hydrodynamic pole with

$$
\omega= \pm \frac{k}{\sqrt{3}}-\frac{i}{8} \frac{\chi}{\epsilon_{0}} k^{2}
$$

and a nonhydrodynamic pole with

$$
\omega=-i \frac{4 \epsilon_{0}}{3 \chi}
$$

\section{b. Vector sector}

From the vector part of Eq. (A14) we get

$$
v_{V}^{i}=\frac{2}{3}\left(\frac{-\epsilon_{0} k^{2}}{-\eta k^{2}+\frac{4}{3} i \omega \epsilon_{0}+\lambda \omega^{2}}\right) h_{V}^{i}
$$

When $k^{2} \rightarrow 0$ we get a hydrodynamic mode

$$
\omega=-\frac{3}{4} i \frac{\eta}{\epsilon_{0}} k^{2}
$$

and a nonhydrodynamic mode

$$
\omega=-\frac{4}{3} i \frac{\epsilon_{0}}{\lambda}+\frac{3}{4} i \frac{\eta}{\epsilon_{0}} k^{2}
$$

We see that the causal FOT formalism recovers the hydrodynamic scalar and vector modes, and also nonhydrodynamic modes in these sectors, with no dynamics at all in the tensor sector.

\section{APPENDIX B: EVALUATION OF INTEGRALS}

The integrals (34) may be evaluated explicitly by going to polar coordinates with $\hat{k}_{l} \hat{p}^{l}=\cos \theta=x$

$$
J=\frac{1}{4 \pi^{2}} \int_{0}^{\infty} d p p^{4} e^{-p / T_{0}} \int_{-1}^{1} \frac{d x}{z-x}=2 \sigma T_{0}^{5} L[z],
$$

where

$$
L[z]=\ln \frac{z+1}{z-1}=2 \sum_{n=0}^{\infty} \frac{1}{(2 n+1)} \frac{1}{z^{2 n+1}}
$$

The radial integral is always the same, while the $x$ integral changes. We have

$$
J^{k}=\chi_{1} \hat{k}^{k}
$$

$$
\begin{aligned}
\chi_{1} & =2 \sigma T_{0}^{5} \int_{-1}^{1} \frac{d x x}{z-x} \\
& =2 \sigma T_{0}^{5}[z L[z]-2]=\frac{4}{3} \sigma T_{0}^{5}\left[\frac{1}{z^{2}}+\frac{3}{5} \frac{1}{z^{4}}+\ldots\right] .
\end{aligned}
$$

To evaluate $J^{j k}$, we write

$$
J^{j k}=\chi_{2}\left[\hat{k}^{j} \hat{k}^{k}-\delta^{j k}\right]+\chi_{3}\left[\hat{k}^{j} \hat{k}^{k}-\frac{1}{3} \delta^{j k}\right] .
$$

Then

$$
\begin{gathered}
\chi_{2}=-\frac{1}{2} J_{j}^{j}=-\frac{1}{2} J=-2 \sigma T_{0}^{5}\left[\frac{1}{z}+\frac{1}{3} \frac{1}{z^{3}}+\ldots\right], \\
\chi_{3}=3 \sigma T_{0}^{5} \int_{-1}^{1} \frac{d x x^{2}}{z-x}, \\
=6 \sigma T_{0}^{5}\left[\frac{1}{2} z^{2} L[z]-z\right]=2 \sigma T_{0}^{5}\left[\frac{1}{z}+\frac{3}{5} \frac{1}{z^{3}}+\ldots\right] .
\end{gathered}
$$

A totally symmetric third order tensor may be written as

$$
J^{i j k}=a \hat{k}^{i} \hat{k}^{j} \hat{k}^{k}+b\left(\hat{k}^{i} \delta^{j k}+\hat{k}^{j} \delta^{k i}+\hat{k}^{k} \delta^{i j}\right) .
$$

A traceless tensor must have $a+5 b=0$, while a totally divergenceless tensor must have $a+3 b=0$. So we may also write 


$$
J^{i j k}=\chi_{4}\left[\hat{k}^{i} \hat{k}^{j} \hat{k}^{k}-\frac{1}{3}\left(\hat{k}^{i} \delta^{j k}+\hat{k}^{j} \delta^{k i}+\hat{k}^{k} \delta^{i j}\right)\right]+\chi_{5}\left[\hat{k}^{i} \hat{k}^{j} \hat{k}^{k}-\frac{1}{5}\left(\hat{k}^{i} \delta^{j k}+\hat{k}^{j} \delta^{k i}+\hat{k}^{k} \delta^{i j}\right)\right] .
$$

Now $J_{j}^{i j}=J^{i}$, so

$$
\chi_{4}=-\frac{3}{2} \chi_{1}=-2 \sigma T_{0}^{5}\left[\frac{1}{z^{2}}+\frac{3}{5} \frac{1}{z^{4}}+\ldots\right]
$$

and then

$$
\begin{aligned}
\chi_{5} & =5 \sigma T_{0}^{5} \int_{-1}^{1} \frac{d x x^{3}}{z-x}, \\
& =5 \sigma T_{0}^{5}\left[z^{3} L[z]-2 z^{2}-\frac{2}{3}\right]=2 \sigma T_{0}^{5}\left[\frac{1}{z^{2}}+\frac{5}{7} \frac{1}{z^{4}}+\ldots\right] .
\end{aligned}
$$

Finally we compute $J^{i j k l}$. Start from

$$
\begin{aligned}
J^{i j k l}= & a \hat{k}^{i} \hat{k}^{j} \hat{k}^{k} \hat{k}^{l} \\
& +b\left(\hat{k}^{i} \hat{k}^{j} \delta^{k l}+\hat{k}^{i} \hat{k}^{k} \delta^{l j}+\hat{k}^{i} \hat{k}^{l} \delta^{j k}+\hat{k}^{j} \hat{k}^{k} \delta^{i l}+\hat{k}^{j} \hat{k}^{l} \delta^{i k}+\hat{k}^{k} \hat{k}^{l} \delta^{i j}\right) \\
& +c\left(\delta^{i j} \delta^{k l}+\delta^{i k} \delta^{l j}+\delta^{i l} \delta^{j k}\right) .
\end{aligned}
$$

We then have

$$
J_{k}^{i j k}=(a+7 b) \hat{k}^{i} \hat{k}^{j}+(b+5 c) \delta^{i j}=J^{i j} .
$$

Then

$$
\begin{aligned}
& a+7 b=\chi_{2}+\chi_{3}, \\
& b+5 c=-\chi_{2}-\frac{1}{3} \chi_{3},
\end{aligned}
$$

so

$$
\begin{aligned}
J^{i j k l}= & \chi_{2}\left[8 \hat{k}^{i} \hat{k}^{j} \hat{k}^{k} \hat{k}^{l}-\left(\hat{k}^{i} \hat{k}^{j} \delta^{k l}+\hat{k}^{i} \hat{k}^{k} \delta^{l j}+\hat{k}^{i} \hat{k}^{l} \delta^{j k}+\hat{k}^{j} \hat{k}^{k} \delta^{i l}+\hat{k}^{j} \hat{k}^{l} \delta^{i k}+\hat{k}^{k} \hat{k}^{l} \delta^{i j}\right)\right] \\
& +\frac{1}{3} \chi_{3}\left[10 \hat{k}^{i} \hat{k}^{j} \hat{k}^{k} \hat{k}^{l}-\left(\hat{k}^{i} \hat{k}^{j} \delta^{k l}+\hat{k}^{i} \hat{k}^{k} \delta^{l j}+\hat{k}^{i} \hat{k}^{l} \delta^{j k}+\hat{k}^{j} \hat{k}^{k} \delta^{i l}+\hat{k}^{j} \hat{k}^{l} \delta^{i k}+\hat{k}^{k} \hat{k}^{l} \delta^{i j}\right)\right] \\
& +\chi_{6}\left[\delta^{i j} \delta^{k l}+\delta^{i k} \delta^{l j}+\delta^{i l} \delta^{j k}-5\left(\hat{k}^{i} \hat{k}^{j} \delta^{k l}+\hat{k}^{i} \hat{k}^{k} \delta^{l j}+\hat{k}^{i} \hat{k}^{l} \delta^{j k}+\hat{k}^{j} \hat{k}^{k} \delta^{i l}+\hat{k}^{j} \hat{k}^{l} \delta^{i k}+\hat{k}^{k} \hat{k}^{l} \delta^{i j}\right)+35 \hat{k}^{i} \hat{k}^{j} \hat{k}^{k} \hat{k}^{l}\right]
\end{aligned}
$$

and

$$
\begin{aligned}
2 \chi_{2}+\frac{4}{3} \chi_{3}+8 \chi_{6} & =2 \sigma T_{0}^{5} \int_{-1}^{1} \frac{d x x^{4}}{z-x} \\
& =2 \sigma T_{0}^{5}\left[z^{4} L[z]-2 z^{3}-\frac{2}{3} z\right],
\end{aligned}
$$

therefore

$$
\chi_{6}=\frac{1}{2} \sigma T_{0}^{5}\left[\frac{1}{2}\left(z^{2}-1\right)^{2} L[z]-z^{3}+\frac{5}{3} z\right]=\frac{4}{15} \sigma T_{0}^{5}\left[\frac{1}{z}+\frac{1}{7} \frac{1}{z^{3}}+\ldots\right]
$$




\section{APPENDIX C: THE $T_{\alpha}^{\mu_{1} \ldots \mu_{n}}$ TENSORS}

In this appendix we evaluate the $T_{\alpha}^{\mu_{1} \ldots \mu_{n}}$ tensors from Eq. (93). The integrals against $f_{\text {eq }}$ may be computed on symmetry, tracelessness and dimensional grounds

$$
\begin{aligned}
T_{0}^{\mu \nu}= & \sigma T_{0}^{4}\left[u^{\mu} u^{\nu}+\frac{1}{3} \Delta^{\mu \nu}\right] \\
T_{1}^{\mu \nu \rho}= & \sigma T_{0}^{4}\left[u^{\mu} u^{\nu} u^{\rho}+\frac{1}{3}\left(\Delta^{\mu \nu} u^{\rho}+\ldots\right)\right], \\
T_{1}^{\mu \nu \rho \sigma}= & 4 \sigma T_{0}^{5}\left[u^{\mu} u^{\nu} u^{\rho} u^{\sigma}+\frac{1}{3}\left(\Delta^{\mu \nu} u^{\rho} u^{\sigma}+\ldots\right)+\frac{1}{15}\left(\Delta^{\mu \nu} \Delta^{\rho \sigma}+\ldots\right)\right], \\
T_{2}^{\mu \nu \rho \sigma}= & \sigma T_{0}^{4}\left[u^{\mu} u^{\nu} u^{\rho} u^{\sigma}+\frac{1}{3}\left(\Delta^{\mu \nu} u^{\rho} u^{\sigma}+\ldots\right)+\frac{1}{15}\left(\Delta^{\mu \nu} \Delta^{\rho \sigma}+\ldots\right)\right], \\
T_{3}^{\mu \nu \rho \sigma \lambda \tau}= & 4 \sigma T_{0}^{5}\left[u^{\mu} u^{\nu} u^{\rho} u^{\sigma} u^{\lambda} u^{\tau}+\frac{1}{3}\left(\Delta^{\mu \nu} u^{\rho} u^{\sigma} u^{\lambda} u^{\tau}+\ldots\right)+\frac{1}{15}\left(\Delta^{\mu \nu} \Delta^{\rho \sigma} u^{\lambda} u^{\tau}+\ldots\right)\right. \\
& \left.+\frac{1}{105}\left(\Delta^{\mu \nu} \Delta^{\rho \sigma} \Delta^{\lambda \tau}+\ldots\right)\right] .
\end{aligned}
$$

[1] P. Romatschke and U. Romatschke, Relativistic Fluid Dynamics In and Out of Equilibrium And Applications to Relativistic Nuclear Collisions (Cambridge University Press, Cambridge, England, 2019).

[2] E. McDonough, The cosmological heavy ion collider: Fast thermalization after cosmic inflation, Phys. Lett. B 809, 135755 (2020).

[3] E. Calzetta and B. L. Hu, Nonequilibrium Quantum Field Theory (Cambridge University Press, Cambridge, England, 2008).

[4] L. Rezzolla and O. Zanotti, Relativistic Hydrodynamics (Oxford University Press, Oxford, 2013).

[5] G.S. Denicol and J. Noronha, Connecting far-fromequilibrium hydrodynamics to resummed transport coefficients and attractors, Nucl. Phys. A1005, 121748 (2021).

[6] M. Strickland, The non-equilibrium attractor for kinetic theory in relaxation time approximation, J. High Energy Phys. 12 (2018) 128.

[7] A. Behtash, C. N. Cruz-Camacho, and M. Martínez, Far-from-equilibrium attractors and nonlinear dynamical systems approach to the Gubser flow, Phys. Rev. D 97, 044041 (2018).

[8] P. Romatschke, Relativistic Fluid Dynamics Far From Local Equilibrium, Phys. Rev. Lett. 120, 012301 (2018).

[9] M. Strickland, J. Noronha, and G. S. Denicol, Anisotropic nonequilibrium hydrodynamic attractor, Phys. Rev. D 97, 036020 (2018).

[10] C. Chattopadhyay and U. Heinz, Hydrodynamics from free-streaming to thermalization and back again, Phys. Lett. B 801, 135158 (2020).
[11] A. Kurkela, W. van der Schee, U. A. Wiedemann, and B. $\mathrm{Wu}$, Early- and Late-Time Behavior of Attractors in Heavy-Ion Collisions, Phys. Rev. Lett. 124, 102301 (2020).

[12] U. Heinz, D. Bazow, G. S. Denicol, M. Martínez, M. Nopoush, J. Noronha, R. Ryblewski, and M. Strickland, Exact solutions of the Boltzmann equation and optimized hydrodynamic approaches for relativistic heavy-ion collisions, Nucl. Part. Phys. Proc. 276, 193 (2016).

[13] M. Martínez, M. McNelis, and U. Heinz, Anisotropic fluid dynamics for Gubser flow, Phys. Rev. C 95, 054907 (2017).

[14] F. S. Bemfica, M. Disconzi, and J. Noronha, Causality and existence of solutions of relativistic viscous fluid dynamics with gravity, Phys. Rev. D 98, 104064 (2018).

[15] L. Cantarutti and E. Calzetta, Dissipative-type theories for Bjorken and Gubser flows, Int. J. Mod. Phys. A 35, 2050074 (2020).

[16] J. L. Anderson and H. R. Witting, A relativistic relaxationtime model for the Boltzmann equation, Physica (Amsterdam) 74, 466 (1974).

[17] J. L. Anderson and H. R. Witting, Relativistic quantum transport coefficients, Physica (Amsterdam) 74, 489 (1974).

[18] P. L. Bhatnagar, E. P. Gross, and M. Krook, A model for collision processes in gases. I. Small amplitude processes in charged and neutral one-component systems, Phys. Rev. 94, 511 (1954).

[19] M. Takamoto and S. I. Inutsuka, The relativistic kinetic dispersion relation: Comparison of the relativistic 
Bhatnagar-Gross-Krook model and Grad's 14-moment expansion, Physica (Amsterdam) 389A, 4580 (2010).

[20] R. Baier, P. Romatschke, D. T. Son, A. O. Starinets, and M. A. Stephanov, Relativistic viscous hydrodynamics, conformal invariance, and holography, J. High Energy Phys. 04 (2008) 100.

[21] P. Romatschke, Retarded correlators in kinetic theory: Branch cuts, poles and hydrodynamic onset transitions, Eur. Phys. J. C 76, 352 (2016).

[22] A. Kurkela and U. A. Wiedemann, Analytic structure of nonhydrodynamic modes in kinetic theory, Eur. Phys. J. C 79, 776 (2019).

[23] W. Florkowski, M. P Heller, and M. Spaliński, New theories of relativistic hydrodynamics in the LHC era, Rep. Prog. Phys. 81, 046001 (2018).

[24] M. P. Heller, A. Serantes, M. Spaliński, V. Svensson, and B. Withers, Convergence of hydrodynamic modes: Insights from kinetic theory and holography, SciPost Phys. 10, 123 (2021).

[25] G. Moore, Stress-stress correlator in $\lambda \phi^{4}$ theory: Poles or a cut?, J. High Energy Phys. 05 (2018) 084.

[26] G. Policastro, D. T. Son, and A. O. Starinets, The Shear Viscosity of Strongly Coupled $N=4$ Supersymmetric Yang-Mills Plasma, Phys. Rev. Lett. 87, 081601 (2001).

[27] D. T. Son and A. O. Starinets, Minkowski-space correlators in AdS/CFT correspondence: Recipe and applications, J. High Energy Phys. 09 (2002) 042.

[28] G. Policastro, D. T. Son, and A. O. Starinets, From AdS/CFT correspondence to hydrodynamics, J. High Energy Phys. 09 (2002) 043.

[29] G. Policastro, D. T. Son, and A. O. Starinets, From AdS/CFT correspondence to hydrodynamics. 2. Sound waves, J. High Energy Phys. 12 (2002) 054.

[30] C. P. Herzog and D. T. Son, Schwinger-Keldysh propagators from AdS/CFT correspondence, J. High Energy Phys. 03 (2003) 046.

[31] P. Kovtun, D. T. Son, and A. O. Starinets, Viscosity in Strongly Interacting Quantum Field Theories from Black Hole Physics, Phys. Rev. Lett. 94, 111601 (2005).

[32] P. K. Kovtun and A. O. Starinets, Quasinormal modes and holography, Phys. Rev. D 72, 086009 (2005).

[33] P. M. Chesler and L. G. Yaffe, Boost invariant flow, black hole formation, and far from-equilibrium dynamics in $N=4$ supersymmetric Yang-Mills theory, Phys. Rev. D 82, 026006 (2010).

[34] B. Withers, Short-lived modes from hydrodynamic dispersion relations, J. High Energy Phys. 06 (2018) 059.

[35] S. Grozdanov, P. K. Kovtun, A. O. Starinetsc, and P. Tadić, The complex life of hydrodynamic modes, J. High Energy Phys. 11 (2019) 097.

[36] W. Israel, Covariant fluid mechanics and thermodynamics: An introduction, in Relativistic Fluid Dynamics, edited by A. M. Anile and Y. Choquet-Bruhat (Springer, New York, 1988).

[37] C. Eckart, The thermodynamics of irreversible processes. III. Relativistic theory of the simple fluid, Phys. Rev. 58, 919 (1940).

[38] L. D. Landau and E. M. Lifshitz, Fluid Mechanics (Pergamon Press, Oxford, 1959).
[39] A. Monnai, Landau and Eckart frames for relativistic fluids in nuclear collisions, Phys. Rev. C 100, 014901 (2019).

[40] W. Hiscock and L. Lindblom, Stability and causality in dissipative relativistic fluids, Ann. Phys. (N.Y.) 151, 466 (1983).

[41] W. A. Hiscock and L. Lindblom, Generic instabilities in first-order dissipative relativistic fluid theories, Phys. Rev. D 31, 725 (1985).

[42] W. A. Hiscock and L. Lindblom, Stability in dissipative relativistic fluid theories, Contemp. Math. 71, 181 (1988).

[43] W. A. Hiscock and L. Lindblom, Non linear pathologies in relativistic heat-conducting fluids, Phys. Lett. 131A, 509 (1988).

[44] T. Olson, Stability and causality in the israel-stewart energy frame theory, Ann. Phys. (N.Y.) 199, 18 (1990).

[45] E. Calzetta, Real relativistic fluids in heavy ion collisions, in Geometric, Algebraic, and Topological Methods for Quantum Field Theory: Proceedings of the 2013 Villa de Leyva Summer School, Villa de Leyva, Colombia, 15-27 July 2013, edited by L. Cano, A. Cardona, H. Ocampo, and A. F. Reyes-Lega (World Scientific, Singapore, 2017); , Real relativistic fluids in heavy ion collisions, arXiv:1310.0841.

[46] A. L. García-Preciante, M. E. Rubio, and O. A. Reula, Generic instabilities in the relativistic Chapman-Enskog heat conduction law, J. Stat. Phys. 181, 246 (2020).

[47] A. L. García-Preciante and O. A. Reula, On the illposedness and stability of the relativistic heat equation, J. Math. Phys. (N.Y.) 61, 051506 (2020).

[48] L. Gavassino, M. Antonelli, and B. Haskell, When the entropy has no maximum: A new perspective on the instability of the first-order theories of dissipation, Phys. Rev. D 102, 043018 (2020).

[49] T. Koide, G. S. Denicol, Ph. Mota, and T. Kodama, Relativistic dissipative hydrodynamics: A minimal causal theory, Phys. Rev. C 75, 034909 (2007).

[50] G. S. Denicol, T. Kodama, T. Koide, and P. Mota, Extensivity of irreversible current and stability in causal dissipative hydrodynamics, J. Phys. G 36, 035103 (2009).

[51] P. Ván, Generic stability of dissipative non-relativistic and relativistic fluids, J. Stat. Mech. (2009) P02054.

[52] P. Ván and T.S. Biró, First order and stable relativistic dissipative hydrodynamics, Phys. Lett. B 709, 106 (2012).

[53] F. S. Bemfica, M. Disconzi, and J. Noronha, Nonlinear causality of general first-order relativistic viscous hydrodynamics, Phys. Rev. D 100, 104020 (2019).

[54] F. S. Bemfica, M. Disconzi, and J. Noronha, Generalrelativistic viscous fluid dynamics, arXiv:2009.11388.

[55] P. Kovtun, First-order relativistic hydrodynamics is stable, J. High Energy Phys. 10 (2019) 034.

[56] R. E. Hoult and P. Kovtun, Stable and causal relativistic Navier-Stokes equations, J. High Energy Phys. 06 (2020) 067.

[57] H. Freistühler, Nonexistence and existence of shock profiles in the Bemfica-Disconzi-Noronha model, Phys. Rev. D 103, 124045 (2021).

[58] A. Pandya and F. Pretorius, Numerical exploration of firstorder relativistic hydrodynamics, Phys. Rev. D 104, 023015 (2021). 
[59] S. Bhattacharyya, V.E. Hubeny, S. Minwalla, and M. Rangamani, Nonlinear fluid dynamics from gravity, J. High Energy Phys. 02 (2008) 045.

[60] A. Jaiswal, Relativistic third-order dissipative fluid dynamics from kinetic theory, Phys. Rev. C 88, 021903(R) (2013).

[61] S. M. Diles, L. A. H. Mamani, A. S. Miranda, and V. T. Zanchin, Third-order relativistic hydrodynamics: Dispersion relations and transport coefficients of a dual plasma, J. High Energy Phys. 05 (2020) 019.

[62] W. Israel, Nonstationary irreversible thermodynamics: A causal relativistic theory, Ann. Phys. (N.Y.) 100, 310 (1976).

[63] W. Israel and J. M. Stewart, Thermodynamics of nonstationary and transient effects in a relativistic gas, Phys. Lett. A 58, 213 (1976).

[64] W. Israel and M. Stewart, Transient relativistic thermodynamics and kinetic theory, Ann. Phys. (N.Y.) 118, 341 (1979).

[65] W. Israel and M. Stewart, On transient relativistic thermodynamics and kinetic theory. II, Proc. R. Soc. A 365, 43 (1979).

[66] W. Israel and J. M. Stewart, Progress in relativistic thermodynamics and electrodynamics of continuous media, in General Relativity and Gravitation, edited by A. Held (Plenum, New York, 1980), Vol. 2, p. 491.

[67] T. Olson and W. Hiscock, Plane steady shock waves in Israel-Stewart fluids, Ann. Phys. (N.Y.) 204, 331 (1990).

[68] D. Pavón, D. Jou, and J. Casas-Vázquez, Heat conduction in relativistic thermodynamics, J. Phys. A 13, L77 (1980).

[69] D. Jou, J. M. Rubi, and J. Casas-Vázquez, Hydrodynamical uctuations in extended irreversible thermodynamics, Physica (Amsterdam) 101A, 588 (1980).

[70] D. Jou, J. Casas-Vázquez, and G. Lebon, Extended Irreversible Thermodynamics (Springer, Berlin, Germany, 2010).

[71] G. S. Denicol, T. Koide, and D. H. Rischke, Dissipative Relativistic Fluid Dynamics: A New Way to Derive the Equations of Motion from Kinetic Theory, Phys. Rev. Lett. 105, 162501 (2010).

[72] B. Betz, G. S. Denicol, T. Koide, E. Molnár, H. Niemi, and D. H. Rischke, Second order dissipative fluid dynamics from kinetic theory, Eur. Phys. J. Web Conf. 13, 07005 (2011).

[73] G. S. Denicol, J. Noronha, H. Niemi, and D. H. Rischke, Origin of the relaxation time in dissipative fluid dynamics, Phys. Rev. D 83, 074019 (2011).

[74] G. S. Denicol, E. Molnár, H. Niemi, and D. H. Rischke, Derivation of fluid dynamics from kinetic theory with the 14 moment approximation, Eur. Phys. J. A 48, 170 (2012).

[75] G. S. Denicol, H. Niemi, E. Molnár, and D. H. Rischke, Derivation of transient relativistic fluid dynamics from the Boltzmann equation, Phys. Rev. D 85, 114047 (2012); 91, 039902(E) (2015).

[76] G. S. Denicol, H. Niemi, I. Bouras, E. Molnár, Z. Xue, D. H. Rischke, and C. Greiner, Solving the heat-flow problem with transient relativistic fluid dynamics, Phys. Rev. D 89, 074005 (2014).

[77] G. S. Denicol and H. Niemi, Derivation of transient relativistic fluid dynamics from the Boltzmann equation for a multi-component system, Nucl. Phys. A904, 369c (2013).

[78] E. Molnár, H. Niemi, G. S. Denicol, and D. H. Rischke, Relative importance of second-order terms in relativistic dissipative fluid dynamics, Phys. Rev. D 89, 074010 (2014).

[79] H. Niemi and G. S. Denicol, How large is the Knudsen number reached in fluid dynamical simulations of ultrarelativistic heavy ion collisions?, arXiv:1404.7327.

[80] M. Strickland, Anisotropic hydrodynamics: Three lectures, Act. Phys. Pol. B 45, 2355 (2014).

[81] M. Strickland, Anisotropic hydrodynamics: Motivation and methodology, Nucl. Phys. A926, 92 (2014).

[82] W. Florkowski, E. Maksymiuk, R. Ryblewski, and L. Tinti, Anisotropic hydrodynamics for mixture of quark and gluon fluids, Phys. Rev. C 92, 054912 (2015).

[83] W. Florkowski, R. Ryblewski, M. Strickland, and L. Tinti, Non-boost-invariant dissipative hydrodynamics, Phys. Rev. C 94, 064903 (2016).

[84] H. Niemi, E. Molnár, and D. H. Rischke, The right choice of moment for anisotropic fluid dynamics, Nucl. Phys. A967, 409 (2017).

[85] D. Bazow, U. Heinz, and M. Strickland, Second-order $(2+1)$-dimensional anisotropic hydrodynamics, Phys. Rev. C 90, 054910 (2014).

[86] E. Molnár, H. Niemi, and D. H. Rischke, Derivation of anisotropic dissipative fluid dynamics from the Boltzmann equation, Phys. Rev. D 93, 114025 (2016).

[87] E. Molnár, H. Niemi, and D. H. Rischke, Closing the equations of motion of anisotropic fluid dynamics by a judicious choice of a moment of the Boltzmann equation, Phys. Rev. D 94, 125003 (2016).

[88] I. S. Liu, I. Müller, and T. Ruggeri, Relativistic thermodynamics of gases, Ann. Phys. (N.Y.) 169, 191 (1986).

[89] R. Geroch and L. Lindblom, Dissipative relativistic fluid theories of divergence type, Phys. Rev. D 41, 1855 (1990).

[90] R. Geroch and L. Lindblom, Causal theories of dissipative relativistic fluids, Ann. Phys. (N.Y.) 207, 394 (1991).

[91] O. A. Reula and G. B. Nagy, On the causality of a dilute gas as a dissipative relativistic fluid theory of divergence type, J. Phys. A 28, 6943 (1995).

[92] L. Lehner, O. A. Reula, and M. E. Rubio, A hyperbolic theory of relativistic conformal dissipative fluids, Phys. Rev. D 97, 024013 (2018).

[93] E. Calzetta, Relativistic fluctuating hydrodynamics, Classical Quantum Gravity 15, 653 (1998).

[94] J. Peralta-Ramos and E. Calzetta, Divergence-type nonlinear conformal hydrodynamics, Phys. Rev. D 80, 126002 (2009).

[95] J. Peralta-Ramos and E. Calzetta, Divergence-type $2+1$ dissipative hydrodynamics applied to heavy-ion collisions, Phys. Rev. C 82, 054905 (2010).

[96] J. Peralta-Ramos and E. Calzetta, Macroscopic approximation to relativistic kinetic theory from a nonlinear closure, Phys. Rev. D 87, 034003 (2013).

[97] N. Mirón-Granese, A. Kandus, and E. Calzetta, Nonlinear fluctuations in relativistic causal fluids, J. High Energy Phys. 07 (2020) 064.

[98] E. Calzetta, Fully developed relativistic turbulence, Phys. Rev. D 103, 056018 (2021). 
[99] A. Das, W. Florkowski, J. Noronha, and R. Ryblewski, Equivalence between first-order causal and stable hydrodynamics and Israel-Stewart theory for boost-invariant systems with a constant relaxation time, Phys. Lett. B 806, 135525 (2020).

[100] S. R. de Groot and P. Mazur, Non-Equilibrium Thermodynamics (Dover, Nueva York, 1984).

[101] L. D. Landau and E. M. Lifshitz, Statistical Physics, Part 1 (Pergamon Press, Oxford, 1980); Part 2 (Pergamon Press, Oxford, 1980).

[102] W. Israel, The relativistic Boltzmann equation, in General Relativity: Papers in Honour of J. L. Synge, edited by L. O'Raifeartaigh (Clarendon Press, Oxford, 1972), p. 201.

[103] G. 't Hooft, A planar diagram theory for strong interactions, Nucl. Phys. B72, 461 (1974).

[104] S. Waeber, A. Schäfer, A. Vuorinen, and L. G. Yaffe, Finite coupling corrections to holographic predictions for hot QCD, J. High Energy Phys. 11 (2015) 087.

[105] G. S. Rocha, G.S. Denicol, and J. Noronha, Novel Relaxation Time Approximation to the Relativistic Boltzmann Equation, Phys. Rev. Lett. 127, 042301 (2021).

[106] E. Calzetta and J. Peralta Ramos, Linking the hydrodynamic and kinetic description of a dissipative relativistic conformal theory, Phys. Rev. D 82, 106003 (2010).

[107] M. Luzum and J.-Y. Ollitrault, Constraining the viscous freeze-out distribution function with data obtained at the BNL relativistic heavy ion collider (RHIC), Phys. Rev. C 82, 014906 (2010).

[108] K. Dusling, G. Moore, and D. Teaney, Radiative energy loss and v(2) spectra for viscous hydrodynamics, Phys. Rev. C 81, 034907 (2010).

[109] S. R. de Groot, W. A. van Leeuwen, and C. G. van Weert, Relativistic Kinetic Theory (North-Holland, Amsterdam, 1980).

[110] J. M. Stewart, Non-Equilibrium Relativistic Kinetic Theory (Springer, New York, 1971).

[111] C. Cercignani and G. Medeiros Kremer, The Relativistic Boltzmann Equation: Theory and Applications (Birkhauser, Basel, 2002).

[112] L. Landau and E. Lifshitz, Course of Theoretical Physics, Vol X: Physical Kinetics (Pergamon, New York, 1981).

[113] A. Behtash, S. Kamata, M. Martínez, T. Schäfer, and V. Skokov, Transasymptotics and hydrodynamization of the Fokker-Planck equation for gluons, Phys. Rev. D 103, 056010 (2021).

[114] R. Liboff, Kinetic Theory (Springer, New York, 2003).

[115] M. Dudyński, Spectral properties of the linearized Boltzmann operator in $L^{p}$ for $1 \leq p \leq \infty$, J. Stat. Phys. 153, 1084 (2013).

[116] L. Luo and H. Yu, Spectrum analysis of the linearized relativistic Landau equation, J. Stat. Phys. 163, 914 (2016).

[117] E. Calzetta, B. L. Hu, and S. A. Ramsey, Hydrodynamic transport functions from quantum kinetic field theory, Phys. Rev. D 61, 125013 (2000).
[118] O. A. Reula and G. B. Nagy, A causal statistical family of dissipative divergence-type fluids, J. Phys. A 30, 1695 (1997).

[119] W. A. Hiscock and L. Lindblom, Linear plane waves in dissipative relativistic fluids, Phys. Rev. D 35, 3723 (1987).

[120] M. Natsuume and T. Okamura, Causal hydrodynamics of gauge theory plasmas from AdS/CFT duality, Phys. Rev. D 77, 066014 (2008).

[121] N. Mirón-Granese and E. Calzetta, Primordial gravitational wave amplification from causal fields, Phys. Rev. D 97, 023517 (2018).

[122] N. Mirón-Granese, Relativistic viscous effects on the primordial gravitational waves spectrum, J. Cosmol. Astropart. Phys. 06 (2021) 008.

[123] A. El, Z. Xu, and C. Greiner, Extension of relativistic dissipative hydrodynamics to third order, Phys. Rev. C 81, 041901(R) (2010).

[124] S. Grozdanov and N. Kaplis, Constructing higher-order hydrodynamics: The third order, Phys. Rev. D 93, 066012 (2016).

[125] C. Chattopadhyay, A. Jaiswal, S. Pal, and R. Ryblewski, Relativistic third-order viscous corrections to the entropy four-current from kinetic theory, Phys. Rev. C 91, 024917 (2015).

[126] R. Biswas, A Dash, N. Haque, S. Pu, and V. Roy, Causality and stability in relativistic viscous non-resistive magnetofluid dynamics, J. High Energy Phys. 10 (2020) 171.

[127] A. K. Panda, A. Dash, R. Biswas, and V. Roy, Relativistic resistive dissipative magnetohydrodynamics from the relaxation time approximation, Phys. Rev. D 104, 054004 (2021).

[128] E. S. Weibel, Spontaneously Growing Transverse Waves in a Plasma Due to an Anisotropic Velocity Distribution, Phys. Rev. Lett. 2, 83 (1959).

[129] E. Calzetta and A. Kandus, A hydrodynamic approach to the study of anisotropic instabilities in dissipative relativistic plasmas, Int. J. Mod. Phys. A 31, 1650194 (2016).

[130] A. Kandus, N. Mirón-Granese, and E. Calzetta, Primordial Weibel Instability, arXiv:2101.03644v2.

[131] M. Maggiore, Gravitational Waves, Vol. 1: Theory and Experiments (Oxford University Press, Oxford, 2008); Vol. 2: Astrophysics and Cosmology (Oxford University Press, Oxford, 2018).

[132] C. Caprini and D. G. Figueroa, Cosmological backgrounds of gravitational waves, Classical Quantum Gravity 35, 163001 (2018).

[133] M. B. Hindmarsh, M. Lüben, J. Lumma, and M. Pauly, Phase transitions in the early universe, SciPost Phys. Lect. Notes 24 (2021).

[134] J. L. Friedman and N. Stergioulas, Rotating Relativistic Stars (Cambridge University Press, Cambridge, England, 2013). 